\title{
Convergence of a particle-in-cell scheme for the spherically symmetric Vlasov-Einstein system
}

\author{
Gerhard Rein \\ Institut für Mathematik, Universität Wien \\ Strudlhofgasse 4, A-1090 Vienna, Austria \\ Thomas Rodewis \\ Nabburger Straße 19, D-81737 Munich, Germany
}

\begin{abstract}
We consider spherically symmetric, asymptotically flat space-times with a collisionless gas as matter model. Many properties of the resulting Vlasov-Einstein system are not yet accessible by purely analytical means. We present a discretized version of this system which is suitable for numerical implementation and is based on the particle-in-cell technique. Convergence of the resulting approximate solutions to the exact solution is proven and error bounds are provided.
\end{abstract}

\section{Introduction}

The properties of space-times filled with matter are of considerable interest in general relativity. The analytical and numerical feasibility as well as the predictions of the models depend to a large extent on the choice of matter model. A model for which considerable progress has been made is the collisionless gas. We consider a large ensemble of particles, all of which are assumed to have the same rest mass, normalized to unity, and to move forward in time. Therefore, their number density $f$ is a non-negative function supported on the mass shell

$$
P M:=\left\{g_{\alpha \beta} p^{\alpha} p^{\beta}=-1, p^{0}>0\right\},
$$

a sub-manifold of the tangent bundle $T M$ of the space-time manifold $M$ with metric $g_{\alpha \beta}$. We use coordinates $\left(t, x^{a}\right)$ with zero shift and corresponding canonical momenta $p^{\alpha}$; Greek indices always run from 0 to 3 , and Latin ones from 1 to 3 . On the mass shell $P M$ the variable $p^{0}$ becomes a function of the remaining variables $\left(t, x^{a}, p^{b}\right)$ :

$$
p^{0}=\sqrt{-g^{00}} \sqrt{1+g_{a b} p^{a} p^{b}} .
$$


The Vlasov-Einstein system which governs the time evolution of such a fully relativistic, self-gravitating collisionless gas now reads

$$
\begin{gathered}
\partial_{t} f+\frac{p^{a}}{p^{0}} \partial_{x^{a}} f-\frac{1}{p^{0}} \Gamma_{\beta \gamma}^{a} p^{\beta} p^{\gamma} \partial_{p^{a}} f=0, \\
G^{\alpha \beta}=8 \pi T^{\alpha \beta}, \\
T^{\alpha \beta}=\int p^{\alpha} p^{\beta} f|g|^{1 / 2} \frac{d p^{1} d p^{2} d p^{3}}{-p_{0}}
\end{gathered}
$$

where $\Gamma_{\beta \gamma}^{\alpha}$ are the Christoffel symbols, $|g|$ denotes the determinant of the metric, $G^{\alpha \beta}$ the Einstein tensor, and $T^{\alpha \beta}$ is the energy-momentum tensor. All physical constants, in particular the speed of light, are set to unity. We refer to [i] for a review of results on this system. Note that the characteristic system

$$
\dot{x}^{a}=p^{a} / p^{0}, \quad \dot{p}^{a}=\Gamma_{\beta \gamma}^{a} p^{\beta} p^{\gamma} / p^{0}
$$

of the Vlasov equation are precisely the geodesic equations in the given metric, written in coordinate time $t$. The characteristic flow leaves the mass shell $P M$ invariant.

Little is known about the behavior of solutions to the Vlasov-Einstein system in full generality. In the present investigation we restrict ourselves to the spherically symmetric and asymptotically flat case. Using Schwarzschild coordinates the metric is taken to be

$$
d s^{2}=-e^{2 \mu(t, r)} d t^{2}+e^{2 \lambda(t, r)} d r^{2}+r^{2}\left(d \theta^{2}+\sin ^{2} \theta d \varphi^{2}\right),
$$

where $t \in \mathbb{R}, r \geq 0, \theta \in[0, \pi], \varphi \in[0,2 \pi]$. Asymptotic flatness is then expressed as

$$
\lim _{r \rightarrow \infty} \lambda(t, r)=\lim _{r \rightarrow \infty} \mu(t, r)=0, \quad t \in \mathbb{R},
$$

and a regular centre is guaranteed by

$$
\lambda(t, 0)=0, \quad t \in \mathbb{R} .
$$

To formulate the system it is convenient to let $x=r(\sin \theta \cos \varphi, \sin \theta \sin \varphi, \cos \theta)$ and, instead of using the corresponding canonical momenta to coordinatize the mass shell over the space-time point $(t, x)$ use frame components

$$
v^{a}=p^{a}+\left(e^{\lambda}-1\right) \frac{x \cdot p}{r} \frac{x^{a}}{r}
$$

from now on $\cdot$ denotes the usual scalar product in $\mathbb{R}^{3}$ and $|v|$ the Euclidean length of $v \in \mathbb{R}^{3}$. With the abbreviation

$$
E:=\sqrt{1+|v|^{2}} \text {, i. e., } p^{0}=e^{-\mu} E
$$

the Vlasov-Einstein system now takes the following form:

$$
\partial_{t} f+e^{\mu-\lambda} \frac{v}{E} \cdot \partial_{x} f-\left(\dot{\lambda} \frac{x \cdot v}{r}+e^{\mu-\lambda} \mu^{\prime} E\right) \frac{x}{r} \cdot \partial_{v} f=0,
$$




$$
\begin{aligned}
& e^{-2 \lambda}\left(2 r \lambda^{\prime}-1\right)+1=8 \pi r^{2} \rho, \\
& e^{-2 \lambda}\left(2 r \mu^{\prime}+1\right)-1=8 \pi r^{2} p,
\end{aligned}
$$

where

$$
\begin{aligned}
& \rho(t, r)=\rho(t, x):=\int E f(t, x, v) d v, \\
& p(t, r)=p(t, x):=\int\left(\frac{x \cdot v}{r}\right)^{2} f(t, x, v) \frac{d v}{E} .
\end{aligned}
$$

By and ' we denote the partial derivative with respect to $t$ and $r$ respectively. The phase space distribution function $f$ is assumed to be spherically symmetric, i. e., $f(t, A x, A v)=f(t, x, v)$ for every $A \in \mathrm{SO}(3)$ and $t \in \mathbb{R}, x, v \in \mathbb{R}^{3}$. It should be noted that while the above system is well-posed is is not the complete VlasovEinstein system: Only the 00 and 11 components of the field equations are written. The 01 component is also used in the sequel; like the also non-trivial 22 and 33 components it follows from the reduced system above, and it reads

$$
\dot{\lambda}=-4 \pi r e^{\lambda+\mu} \jmath,
$$

where

$$
\jmath(t, r)=\jmath(t, x):=\int \frac{x \cdot v}{r} f(t, x, v) d v .
$$

Due to the symmetry the field equations have no radiative degrees of freedom, and the solution is determined by an initial condition

$$
f(0, x, v)=\stackrel{\circ}{f}(x, v) .
$$

The initial data are taken to be spherically symmetric, non-negative, continuously differentiable, compactly supported, and such that

$$
\int_{|y| \leq r} \int \stackrel{\circ}{f}(y, v) d v d y<\frac{r}{2}, r \geq 0 .
$$

The latter condition rules out trapped surfaces at $t=0$. We briefly discuss the main analytical results on this system. In [13] it was shown that each initial datum as above launches a unique smooth solution for which all derivatives which appear in the system exist and are continuous. The solution can be extended in time as long as $\rho$ remains bounded. For small initial data it is then shown that a global, geodesically complete solution results which decays to flat Minkowski space for $t \rightarrow \infty$. On the other hand, it is shown in [19] that large data will lead to a singularity, and in [17] it is shown that the first such singularity will be at the centre. The main open problem is whether large data still lead to solutions which are global in Schwarzschild time; if so this would imply the cosmic censorship hypotheses. This problem was investigated by numerical simulation in 18. Given $\stackrel{\circ}{f}$ as above the system was solved numerically with initial datum $A f$ where the amplitude $A>0$ was slowly increased from small 
to large values. There was no indication that the solutions might blow up in finite Schwarzschild time, and there existed a critical amplitude $A_{*}$ such that for $A<A_{*}$ the solution dispersed while for $A>A_{*}$ a black hole with mass $M(A)$ seemed to form. The system exhibited exclusively the so-called type I behavior: $\lim _{A \rightarrow A_{*}+} M(A)=M_{*}>0$. This is in sharp contrast to results for the same sort of numerical experiment with different matter models which exhibit type II behavior, $M_{*}=0$. The findings of [18] are confirmed in [8] so that considerable evidence seems to indicate that cosmic censorship holds for the Vlasov-Einstein system. One should note, however, that in [24, 25, 26, 27] the formation of naked singularities was reported in a numerical simulation of the Vlasov-Einstein system with axial symmetry. On the other hand, certain numerical findings which were claimed there for the Vlasov-Poisson system which is the Newtonian limit of the Vlasov-Einstein system contradict known analytical results.

Given the significance of these questions it is desirable to have a rigorous mathematical foundation for the numerical scheme which is used, that is, to show that the solutions of some appropriately discretized version of the system converge to solutions of the Vlasov-Einstein system if the parameters of the discretization vary appropriately, and to give corresponding error bounds. The aim of the present paper is to carry out this program for the scheme used in [18], a so-called particle-in-cell scheme. This scheme is discussed in detail in the next section, the basic set-up being as follows: The support of the initial datum is split into small cells. In each cell a point is chosen which carries a weight representing the integral of $f$ over this cell. These particles are smeared out in space by a hat function. From this approximation for $f$ approximations for the source terms in the field equations and thus approximations for the fields can be determined. With these we enter into the characteristic system of the Vlasov equation and propagate the particles by one time step. Then the process is repeated. This sort of numerical scheme is well known in plasma physics and in astrophysics where it is used to simulate the Vlasov-Maxwell or the Vlasov-Poisson system. For convergence results for these systems we refer to [ 由, 5, 6, 28, 29] and in particular to [22] where the spherically symmetric Vlasov-Poisson system is considered. General background on such schemes can be found in [2, 3].

For the present system particular difficulties arise due to the fact that the source terms in the field equations contribute to the fields also in a pointwise sense; as opposed to the Vlasov-Poisson and Vlasov-Maxwell systems they are not necessarily integrated in space(time). This lack of smoothing effect of the field equations which is apparent for example in (1.8) causes considerable analytical as well as numerical complications, and the analysis of a numerical scheme must be based on a careful analysis of the analytical properties of the solutions, which was initiated in [13], cf. also [11]. The paper proceeds as follows: In the next section we first collect some additional information on the Vlasov-Einstein system which is needed in the sequel. Then we state the discretized version of our system and the main result. At this stage we only discretize in phase space, thereby reducing the system to a system of ordinary differential equations for the particle positions and other relevant quantities. The proofs of our convergence 
results and error estimates are carried out in Section 3 in a series of lemmas. In Section 4 we discuss the question of how to discretize the system also in time. Since our numerical findings coincide with those reported in [17] and [8] we do not include them here. An open problem is to analyze the role which steady states of the system play in explaining the observed type I behavior. This will be the topic of a separate numerical investigation for which the present paper sets the theoretical stage.

We conclude this introduction with some further references to the literature. In 14] and 20] it was shown that solutions of the Vlasov-Einstein system converge to solutions of the Vlasov-Poisson system in the Newtonian limit. The latter system is much better understood than the Vlasov-Einstein system, in particular, there exists an existence result for global smooth solutions to general initial data, cf. 9, 7, 23. Another important feature of the Vlasov-Einstein system as stated above is that it possesses a large family of steady states, cf. [15, 16, 12. All the results mentioned so far refer to the asymptotically flat case which is characterized by the boundary condition (1.1) and from a physics point of view represents an isolated system such as a galaxy or globular cluster in an otherwise empty universe. There exists also a growing number of results on the cosmological case of the Vlasov-Einstein system, and we refer to [1] for a discussion of and references to these results. Finally we mention that the results of the present paper constitute the major part of the second author's PhD thesis [21].

\section{The semi-discretized approximation-main result}

An initial datum $\stackrel{\circ}{f}$ as specified above launches a continuously differentiable and spherically symmetric solution $f$. Let $[0, T]$ denote any time interval on which this solution exists. For numerical investigations it is important to make use of the spherical symmetry in such a way as to reduce the number of independent variables. We introduce

$$
r:=|x|, w:=\frac{x \cdot v}{r}, L=x^{2} v^{2}-(x \cdot v)^{2}=|x \times v|^{2}
$$

so that

$$
E=\sqrt{1+w^{2}+L / r^{2}}
$$

It can be shown that $f$ must be of the form

$$
f(t, x, v)=f(t, r, w, L) .
$$

Instead of writing the Vlasov equation in these variables we write down its characteristic system which is equivalent but more relevant for what follows:

$$
\dot{r}=e^{\mu-\lambda} \frac{w}{E},
$$




$$
\begin{aligned}
\dot{w} & =-\dot{\lambda} w-e^{\mu-\lambda} \mu^{\prime} E+e^{\mu-\lambda} \frac{L}{r^{3} E}, \\
\dot{L} & =0 .
\end{aligned}
$$

Note that the quantity $L$, the modulus of angular momentum squared, is conserved along characteristics so that the characteristic system is essentially two dimensional. The field equations (1.4), (1.5), (1.8) remain unaffected by the above change of variables, and the source terms can be written as

$$
\begin{aligned}
& \rho(t, r)=\frac{\pi}{r^{2}} \int_{-\infty}^{\infty} \int_{0}^{\infty} E f(t, r, w,) d L d w, \\
& p(t, r)=\frac{\pi}{r^{2}} \int_{-\infty}^{\infty} \int_{0}^{\infty} \frac{w^{2}}{E} f(t, r, w, L) d L d w, \\
& \jmath(t, r)=\frac{\pi}{r^{2}} \int_{-\infty}^{\infty} \int_{0}^{\infty} w f(t, r, w, L) d L d w .
\end{aligned}
$$

In the sequel we will use both $(x, v)$ and $(r, w, L)$ in our discussion, but the numerical scheme will be formulated in the latter coordinates. Let us denote by $(X, V)(s, t, x, v)$ or $(R, W, L)(s, t, r, w, L)$ the solution of the characteristic system which at time $s=t$ takes the value $(x, v) \in \mathbb{R}^{6}$ or $(r, w, L) \in[0, \infty[\times \mathbb{R} \times[0, \infty[$. Then (by abuse of notation)

$$
f(t, x, v)=\stackrel{\circ}{f}((X, V)(0, t, x, v))=\stackrel{\circ}{f}((R, W, L)(0, t, r, w, L))=f(t, r, w, L) .
$$

It is important that the field equations can be solved explicitly for $\lambda$ and $\mu$ and their relevant derivatives. Using (1.2) the field equation (1.4) can be integrated to yield

$$
e^{-2 \lambda(t, r)}=1-\frac{2 m(t, r)}{r}
$$

where

$$
m(t, r):=4 \pi \int_{0}^{r} s^{2} \rho(t, s) d s
$$

the right hand side of (2.8) is positive initially by assumption on $\stackrel{\circ}{\text { and remains so }}$ on the existence interval of the solution. It is worthwhile to note that $m(t, \infty)$ is a conserved quantity of the system, the ADM mass. Another conserved quantity, related to the conservation of the number of particles, is

$$
\iint e^{\lambda(t, r)} f(t, x, v) d v d x
$$

Next we solve (1.5) for $\mu^{\prime}$,

$$
\mu^{\prime}(t, r)=e^{2 \lambda(t, r)}\left(\frac{m(t, r)}{r^{2}}+4 \pi r p(t, r)\right),
$$

and using (1.1) this is integrated to give

$$
\mu(t, r)=-\int_{r}^{\infty} \mu^{\prime}(t, s) d s
$$


From (1.4) we also have

$$
\lambda^{\prime}(t, r)=e^{2 \lambda(t, r)}\left(-\frac{m(t, r)}{r^{2}}+4 \pi r \rho(t, r)\right)
$$

which will become relevant shortly. Obviously, $\lambda \geq 0, \mu \leq 0$, and by adding (2.11) and (2.13) and observing the boundary condition (1.1), $\lambda+\mu \leq 0$. As noted above, $f$ is constant along characteristics, but the characteristic flow is not volume preserving as can be seen from the factor $e^{\lambda}$ in (2.10). This complication is the price we pay for writing the system in non-canonical momentum variables which make the explicit representations of the metric quantities in terms of the source terms possible and greatly simplify the Vlasov equation. We need to know how integrals of $f$ over pieces of phase space evolve:

Lemma 2.1 Let $Z(\cdot, t, z)=(X, V)(\cdot, t, x, v)$ denote the solution of the characteristic system of $(1.3)$ with $Z(t, t, z)=z \in \mathbb{R}^{6}$. Then

$$
\operatorname{det} \partial_{z} Z(s, t, z)=e^{\lambda(t, x)-\lambda(s, X(s, t, z))} .
$$

Let $A \subset \mathbb{R}^{6}$ be measurable and $A(t):=Z(t, 0, A)$. Then we have for the solution $f$ and any continuous function $g$,

$$
\int_{A(t)} g(t, z) f(t, z) d z=\int_{A} g(t, Z(t, 0, z)) \stackrel{f}{f}(z) e^{\lambda(0, r)-\lambda(t, R(t, 0, z))} d z
$$

and

$$
\frac{d}{d t} \int_{A(t)} f(t, z) d z=-\int_{A(t)} f(t, z)\left(\dot{\lambda}(t, r)+\dot{R}(t, 0, Z(0, t, z)) \lambda^{\prime}(t, r)\right) d z .
$$

Proof. The first assertion follows from Liouville's Theorem, and the rest follows by a change of variables and straight forward computation.

Since we will not be too careful in distinguishing between a symmetric subset $A \subset \mathbb{R}^{3} \times \mathbb{R}^{3}$ and the subset $\widetilde{A}$ of $(r, w, L)$-space which describes $A$, that is

$$
A=\left\{(x, v) \in \mathbb{R}^{6} \mid\left(|x|, x \cdot v /|x|,|x \times v|^{2}\right) \in \widetilde{A}\right\},
$$

we note that

$$
\operatorname{meas}(A)=4 \pi^{2} \operatorname{meas}(\widetilde{A}) \text {. }
$$

This follows from $d v=\pi r^{-2} d w d L$ and $d x=4 \pi r^{2} d r$.

We make the following additional assumption on the initial datum $f:$ With respect to the $(r, w, L)$-variables,

$$
\operatorname{supp} \stackrel{\circ}{\mathrm{f}} \subset\left[r_{\min }, r_{\max }\right] \times\left[w_{\min }, w_{\max }\right] \times\left[L_{\min }, L_{\max }\right]
$$

with constants $0<r_{\min }<r_{\max }, w_{\min }<w_{\max }, 0<L_{\min }<L_{\max }$. Since $L$ is conserved along particle trajectories, the particles remain away from zero in space as long as their momenta $v$ remain bounded. This assumption, which was also 
made in [22], avoids problems arising from particles at the origin where our coordinates $(r, w, L)$ are not suitable. In [21] it is shown for the Vlasov-Poisson system how this restriction can be avoided by switching to Cartesian coordinates in the neighborhood of the origin, but we prefer to avoid at least this technical complication. In passing we note that the numerical investigations in [18, 8] considered data satisfying this restriction.

We are now ready to formulate the

Semi-discretized approximation: We decompose the support of $f$ in $(r, w, L)$-space or a supset of the form (2.14) into disjoint, connected, and measurable sets $A_{n}, n \in\{1,2, \ldots, N\}$, with

$$
\operatorname{diam}\left(A_{n}\right) \leq \varepsilon, \operatorname{meas}\left(A_{n}\right) \geq \frac{1}{100} \varepsilon^{3}, n \in\{1,2, \ldots, N\},
$$

where the fineness $0<\varepsilon \ll 1$ is a small parameter; the factor $1 / 100$ can be replaced by any positive constant which is then kept fixed. In each cell $A_{n}$ we fix a point $\left(r_{n}, w_{n}, L_{n}\right) \in A_{n}$ and define

$$
\left(R_{n}, W_{n}, L_{n}\right)(t):=(R, W, L)\left(t, 0,\left(r_{n}, w_{n}, L_{n}\right)\right),
$$

the corresponding solutions of the characteristic system (2.2), (2.3), 2.4). The cell in $(x, v)$-space which corresponds to $A_{n}$ is denoted by the same symbol. Moreover, we define

$$
A_{n}(t):=Z\left(t, 0, A_{n}\right), \quad M_{n}(t):=\int_{A_{n}(t)} f(t, z) d z ;
$$

note that Lemma 2.1 tells us how $M_{n}$ evolves.

Our aim is to obtain approximations to $R_{n}(t), W_{n}(t), M_{n}(t)$ as solutions of an appropriately set-up system of ordinary equations. Throughout the paper approximating quantities will be denoted with a bar. Suppose we have $N$ points in $(r, w, L)$-space with coordinates

$$
\begin{aligned}
\bar{R} & =\left(\bar{R}_{1}, \ldots, \bar{R}_{N}\right) \in \mathbb{R}_{+}^{N}, \\
\bar{W} & =\left(\bar{W}_{1}, \ldots, \bar{W}_{N}\right) \in \mathbb{R}^{N}, \\
\bar{L} & =\left(\bar{L}_{1}, \ldots, \bar{L}_{N}\right) \in \mathbb{R}_{+}^{N},
\end{aligned}
$$

carrying weights

$$
\bar{M}=\left(\bar{M}_{1}, \ldots, \bar{M}_{N}\right) \in \mathbb{R}_{+}^{N} .
$$

From this information we generate approximations for the source terms at any $r \geq 0$ : Following (2.1) we abbreviate

$$
\bar{E}_{n}:=\bar{E}\left(\bar{R}_{n}, \bar{W}_{n}, \bar{L}_{n}\right):=\sqrt{1+\bar{W}_{n}^{2}+\bar{L}_{n} / \bar{R}_{n}^{2}}
$$

and define

$$
\bar{\rho}(r, \bar{R}, \bar{W}, \bar{L}, \bar{M}):=\frac{1}{4 \pi r^{2} \delta} \sum_{n=1}^{N} \bar{E}_{n} \bar{M}_{n} \chi_{\delta}\left(r-\bar{R}_{n}\right),
$$




$$
\begin{aligned}
\bar{p}(r, \bar{R}, \bar{W}, \bar{L}, \bar{M}) & :=\frac{1}{4 \pi r^{2} \delta} \sum_{n=1}^{N} \frac{\bar{W}_{n}^{2}}{\bar{E}_{n}} \bar{M}_{n} \chi_{\delta}\left(r-\bar{R}_{n}\right), \\
\bar{\jmath}(r, \bar{R}, \bar{W}, \bar{L}, \bar{M}) & :=\frac{1}{4 \pi r^{2} \delta} \sum_{n=1}^{N} \bar{W}_{n} \bar{M}_{n} \chi_{\delta}\left(r-\bar{R}_{n}\right) .
\end{aligned}
$$

Here the hat function $\chi_{\delta}$ which is used to smear out the particles is defined by

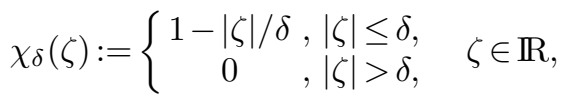

where $0<\delta \ll 1$ is another small parameter; the necessary relations between $\delta$ and $\varepsilon$ will be specified shortly. Suppressing the variables $\bar{R}, \bar{W}, \bar{L}, \bar{M}$ for the moment we can now define approximations for the various quantities appearing on the right hand side of the characteristic system etc.:

$$
\begin{aligned}
\bar{m}(r) & :=4 \pi \int_{0}^{r} s^{2} \bar{\rho}(s) d s, \\
e^{-2 \bar{\lambda}(r)} & :=1-\frac{2 \bar{m}(r)}{r}, \\
\bar{\mu}^{\prime}(r) & :=e^{2 \bar{\lambda}(r)}\left(\frac{\bar{m}(r)}{r^{2}}+4 \pi r \bar{p}(r)\right), \\
\bar{\mu}(r) & :=-\int_{r}^{\infty} \bar{\mu}^{\prime}(s) d s, \\
\bar{\lambda}^{\prime}(r) & :=e^{2 \bar{\lambda}(r)}\left(-\frac{\bar{m}(r)}{r^{2}}+4 \pi r \bar{\rho}(r)\right), \\
\bar{\lambda}(r) & :=-4 \pi r e^{\bar{\lambda}(r)+\bar{\mu}(r)} \bar{\jmath}(r) .
\end{aligned}
$$

The approximations $\bar{R}_{n}(t), \bar{W}_{n}(t), \bar{M}_{n}(t)$ to the true quantities $R_{n}(t), W_{n}(t)$, $M_{n}(t)$ are now defined as the solutions of of the following autonomous system of $3 N$ ordinary differential equations:

$$
\begin{aligned}
\dot{\bar{R}}_{n} & =e^{(\bar{\mu}-\bar{\lambda})\left(\bar{R}_{n}\right)} \frac{\bar{W}_{n}}{\bar{E}_{n}} \\
\dot{\bar{W}}_{n} & =e^{(\bar{\mu}-\bar{\lambda})\left(\bar{R}_{n}\right)} \frac{L_{n}}{\bar{R}_{n}^{3} \bar{E}_{n}}-\bar{\lambda}\left(\bar{R}_{n}\right) \bar{W}_{n}-e^{(\bar{\mu}-\bar{\lambda})\left(\bar{R}_{n}\right)} \bar{\mu}^{\prime}\left(\bar{R}_{n}\right) \bar{E}_{n}, \\
\dot{\bar{M}}_{n} & =-\left(\overline{\dot{\lambda}}\left(\bar{R}_{n}\right)+e^{(\bar{\mu}-\bar{\lambda})\left(\bar{R}_{n}\right)} \frac{\bar{W}_{n}}{\bar{E}_{n}} \bar{\lambda}^{\prime}\left(\bar{R}_{n}\right)\right) \bar{M}_{n}
\end{aligned}
$$

with initial conditions $\left(\bar{R}_{n}, \bar{W}_{n}\right)(0)=\left(r_{n}, w_{n}\right)$ and $\bar{M}_{n}(0)=M_{n}(0), n=1, \ldots, N$. Clearly, we set $\bar{L}_{n}=L_{n}$. Moreover, the dependence of $\bar{\lambda}, \bar{\mu}$, and their various derivatives on the coordinates and weights of all the other particles has been suppressed in the notation above.

Given a solution $\bar{R}(t), \bar{W}(t), \bar{M}(t)$ of the above discretized system it is suggestive to abuse our notation as follows:

$$
\bar{\rho}(t, r):=\bar{\rho}(r, \bar{R}(t), \bar{W}(t), \bar{M}(t), \bar{L}),
$$


and analogously for all the other source terms, metric coefficients, and their various derivatives. Note that $\overline{\dot{\lambda}}$ is just a name. In general $\overline{\dot{\lambda}} \neq \partial_{t} \bar{\lambda}$, whereas $\bar{\mu}^{\prime}=\partial_{r} \bar{\mu}$ and $\bar{\lambda}^{\prime}=\partial_{r} \bar{\lambda}$. Nevertheless, we will use the notation $\overline{\dot{\lambda}}$ because we will compare $\overline{\dot{\lambda}}$ to $\dot{\lambda}$ during the convergence proof.

Before we state the main result we specify certain quantities which we use to control the errors. By $\|\cdot\|_{\infty}$ we denote the $L^{\infty}$ norm with respect to $r \in[0, \infty[$. For the characteristics we define

$$
\begin{aligned}
\|R(t)-\bar{R}(t)\| & :=\max \left\{\left|R_{n}(s)-\bar{R}_{n}(s)\right| \mid n \in\{1, \ldots, N\}, s \in[0, t]\right\}, \\
\|W(t)-\bar{W}(t)\| & :=\max \left\{\left|W_{n}(s)-\bar{W}_{n}(s)\right| \mid n \in\{1, \ldots, N\}, s \in[0, t]\right\}, \\
\|M(t)-\bar{M}(t)\| & :=\varepsilon^{-3} \max \left\{\left|M_{n}(s)-\bar{M}_{n}(s)\right| \mid n \in\{1, \ldots, N\}, s \in[0, t]\right\} ;
\end{aligned}
$$

since the weights $M_{n}$ are of order $\mathrm{O}\left(\varepsilon^{3}\right)$ we have inserted a suitable factor. We are now able to state the main result:

Theorem 2.2 Assume in addition that $\stackrel{\circ}{f} \in C^{2}\left(\mathbb{R}^{6}\right)$ so that the solution has the same regularity with respect to $x$ and $v$. Then there exist constants $C_{1}>0$ and $\mathrm{C}_{2}>0$ depending only on the approximated solution $f$ restricted to the time interval $[0, T]$ such that the following holds: If $0<\varepsilon \leq \delta \leq C_{1}$ then any solution of the discretized system stated above exists on $[0, T]$ and satisfies the following estimates for $t \in[0, T]$ :

$$
\begin{gathered}
\|R(t)-\bar{R}(t)\|+\|W(t)-\bar{W}(t)\|+\|M(t)-\bar{M}(t)\| \leq C\left(\delta^{2}+\frac{\varepsilon}{\delta}\right), \\
\|\lambda(t)-\bar{\lambda}(t)\|_{\infty}+\|\mu(t)-\bar{\mu}(t)\|_{\infty}+\|m(t)-\bar{m}(t)\|_{\infty} \leq C\left(\delta^{2}+\frac{\varepsilon}{\delta}\right), \\
\|\rho(t)-\bar{\rho}(t)\|_{\infty}+\|p(t)-\bar{p}(t)\|_{\infty}+\|\jmath(t)-\bar{\jmath}(t)\|_{\infty} \leq C\left(\delta+\frac{\varepsilon}{\delta^{2}}\right) .
\end{gathered}
$$

Note that the error estimates for the source terms are one order worse than the other estimates; this is no coincidence.

Remark. Strictly speaking there exists in the literature no proof for the assertion that the regularity assumption $\stackrel{\circ}{f} \in C^{2}\left(\mathbb{R}^{6}\right)$ will propagate to the solution. However, $\partial_{x_{i}} f$ will satisfy a system of differential equations which is obtained from the Vlasov-Einstein system by differentiating the various equations accordingly, and this system will be linear in $\partial_{x_{i}} f$ so that this derivative will be $C^{1}$ on the existence interval of $f$. Turning this into a proof would be a lengthy exercise. A version of our theorem without the additional regularity assumption is also possible. However, we then no longer obtain error bounds for the source terms, and instead of the regularity assumption an a-priori bound of the approximating source terms, uniform in $\varepsilon$ and $\delta$ has to be made which can be monitored during a run-time situation. Since the statement of this second, weaker form of our result requires more technical preparation we postpone it to the next section. 


\section{The semi-discretized approximation-proofs}

First we need to introduce some further information on the solutions of the Vlasov-Einstein system. From [13] we know that any initial datum $\stackrel{0}{f} \in C^{1}\left(\mathbb{R}^{6}\right)$ as specified above launches a unique solution $f$. Let $[0, T]$ be any time interval on which this solution exists. Then $f \in C^{1}\left([0, T] \times \mathbb{R}^{6}\right)$ with respect to $(x, v)$ and

$$
\rho, p, \jmath \in C^{1}\left([0, T] \times\left[0, \infty[), m, \lambda, \mu \in C^{2}([0, T] \times[0, \infty[)\right.\right.
$$

as functions of $t$ and $r$. This solution together with the time interval are now kept fixed, and we will need the following bounds:

Lemma 3.1 There is a constant $D \geq 1$ such that for all $t \in[0, T]$ and $r \geq 0$,

$$
\begin{aligned}
& \rho(t, r), p(t, r),|\jmath(t, r)|, e^{2 \lambda(t, r)},|\dot{\lambda}(t, r)|,\left|\lambda^{\prime}(t, r)\right|, \\
& |\mu(t, r)|,\left|\mu^{\prime}(t, r)\right|,\left|\dot{\lambda}^{\prime}(t, r)\right|,\left|\lambda^{\prime \prime}(t, r)\right|,\left|\mu^{\prime \prime}(t, r)\right| \leq D,
\end{aligned}
$$

and for all $t \in[0, T]$ and $(r, w, L) \in \operatorname{supp} \stackrel{\circ}{\text {, }}$

$$
R(t, 0, r, w, L), \frac{1}{R(t, 0, r, w, L)},|\dot{R}(t, 0, r, w, L)|,|W(t, 0, r, w, L)| \leq D .
$$

Moreover, D can be chosen such that

$$
\varepsilon^{-3} M_{n}(t) \leq D, n=1, \ldots, N,
$$

for any discretization of $\operatorname{supp} \stackrel{\circ}{f}$ as specified above.

Proof. The right hand side of (2.2) is bounded by 1 , and hence the source terms vanish outside the light cone, that is for all $(t, r)$ with $r \geq r_{\max }+t$. Together with the regularity of the solution this implies the first set of estimates. Since $V(t, 0, z)$ is uniformly bounded for $t \in[0, T]$ and $z \in \operatorname{supp} f$ and $L$ is conserved we get a lower bound on $R$. Finally, by Lemma 2.1,

$$
M_{n}(t)=\int_{A_{n}(t)} f(t, z) d z \leq \int_{A_{n}} \stackrel{\circ}{f}(z) e^{\lambda(0, r)} d z \leq\left\|\stackrel{\circ}{f} e^{\lambda(0)}\right\|_{\infty} \operatorname{meas}\left(A_{n}\right),
$$

and the proof is complete.

All the functions on the right hand side of (2.17), (2.18), (2.19) are Lipschitzcontinuous in the unknowns. Let $\left[0, T_{\varepsilon, \delta}\right]$ denote the maximal interval on which the approximate solution exists and satisfies the bounds

$$
\varepsilon^{-3} \bar{M}_{n}(t), \bar{R}_{n}(t), \frac{1}{\bar{R}_{n}(t)},\left|\bar{W}_{n}(t)\right|,\left\|e^{2 \bar{\lambda}(t)}\right\|_{\infty} \leq 2 D, n=1, \ldots, N .
$$

Here $D$ is the constant used in Lemma 3.1. It must be emphasized that $T_{\varepsilon, \delta}$ depends on the particular discretization of the support of $\stackrel{\circ}{f}$ and not just on $\varepsilon$ and $\delta$.

We can now formulate the weaker version of our main result which does not require the additional regularity of the approximated solution: 
Theorem 3.2 Let a family of approximating solutions parameterized by $\varepsilon$ and $\delta$ (or a sequence of such) be given and assume that for some constant $C^{\star}>0$,

$$
\bar{\rho}(t) \leq C^{\star}, 0<\varepsilon \leq \delta \leq \frac{1}{4 D}, t \in\left[0, T_{\varepsilon, \delta}\right] .
$$

Then there exist constants $C_{1}>0$ and $C_{2}>0$ depending on the restriction of the approximated solution $f$ to the time interval $[0, T]$ and on $C^{*}$ such that the following holds: If $0<\varepsilon \leq \delta \leq C_{1}$ then any solution of the discretized system stated above exists on $[0, T]$ and satisfies the following estimates for $t \in[0, T]$ :

$$
\begin{gathered}
\|R(t)-\bar{R}(t)\|+\|W(t)-\bar{W}(t)\|+\|M(t)-\bar{M}(t)\| \leq C\left(\delta+\frac{\varepsilon}{\delta}\right), \\
\|\lambda(t)-\bar{\lambda}(t)\|_{\infty}+\|\mu(t)-\bar{\mu}(t)\|_{\infty}+\|m(t)-\bar{m}(t)\|_{\infty} \leq C\left(\delta+\frac{\varepsilon}{\delta}\right) .
\end{gathered}
$$

Note that in a run-time situation the assumption on the boundedness of $\bar{\rho}$ can be monitored by looking at the numerical data.

Throughout the rest of this section we fix discretization parameters

$$
0<\varepsilon \leq \delta \leq \frac{1}{4 D} .
$$

The lower bound on meas $\left(A_{n}\right), n=1, \ldots, N$, which is to hold for any discretization implies that

$$
N \varepsilon^{3} \leq C ;
$$

as in all the estimates which follow $C$ denotes a positive constant which may depend on $D, C^{*}$, or the restriction of $f$ on $[0, T]$. We assume that the assumptions of Theorem 2.2 or Theorem 3.2 hold. In the situation of Theorem 2.2 we redefine $T_{\varepsilon, \delta}$ such that $\left[0, T_{\varepsilon, \delta}\right]$ is the maximal interval on which the estimate

$$
\|\bar{\rho}(t)\|_{\infty} \leq 2 D
$$

holds in addition to those stated in (3.1). In the arguments which follow there is almost no need to distinguish between Theorem 2.2 and Theorem 3.2. The only difference is the bound on $\bar{\rho}: \bar{\rho}(t, r) \leq 2 D$ for $t \in\left[0, T_{\varepsilon, \delta}\right]$ in case of Theorem 2.2 and $\bar{\rho}(t, r) \leq C^{\star}$ for $t \in\left[0, T_{\varepsilon, \delta}\right]$ in case of Theorem 3.2.

We will use the following abbreviation for the index set of the decomposition of $\operatorname{supp} f$ :

$$
\mathcal{N}:=\{1,2, \ldots, N\} .
$$

We start our chain of auxiliary results by collecting some information on our hat function $\chi_{\delta}$ :

Lemma 3.3 Let $\delta>0$ and define

$$
\chi(\zeta):=\frac{1}{\delta} \int_{-\infty}^{\zeta} \chi_{\delta}(\xi) d s, \zeta \in \mathbb{R} .
$$


Then

$$
|\chi(\zeta)-\chi(\xi)| \leq \frac{1}{\delta}|\zeta-\xi|, \quad 0 \leq \chi(\zeta) \leq 1, \zeta, \xi \in \mathbb{R},
$$

and for $a, b, \zeta \in \mathbb{R}$ with $|a-\zeta| \geq|a-b|+\delta$,

$$
\chi(\zeta-a)=\chi(\zeta-b) .
$$

The proof is straight forward. Next we collect some additional information on the characteristic flow of the Vlasov-Einstein system. As before, we often abbreviate $z=(x, v) \in \mathbb{R}^{3} \times \mathbb{R}^{3}$ and $Z=(X, V)$ accordingly.

Lemma 3.4 For all characteristics starting in the support of $\stackrel{\circ}{f}$ and all $t \in[0, T]$,

$$
\left|Z\left(t, 0, z_{1}\right)-Z\left(t, 0, z_{2}\right)\right| \leq C\left|z_{1}-z_{2}\right|,
$$

in particular, for $A \subset \operatorname{supp} \stackrel{\circ}{f} \subset \mathbb{R}^{6}$ and $A(t):=Z(t, 0, A)$,

$$
\operatorname{diam}(A(t)) \leq C \operatorname{diam}(A) .
$$

Also

$$
\begin{aligned}
\mid(R, W)\left(t, 0, r_{1}, w_{1}, L_{1}\right)- & (R, W)\left(t, 0, r_{2}, w_{2}, L_{2}\right) \mid \\
\leq & C\left(\left|r_{1}-r_{2}\right|+\left|w_{1}-w_{2}\right|+\left|L_{1}-L_{2}\right|\right) .
\end{aligned}
$$

Proof. The first estimate is included in [11, Prop. 2.2], with the bounds from Lemma 3.1 this implies the third estimate; note that $1 / R(t)$ is bounded.

We now start establishing bounds on the approximating solutions; recall that constants $C$ may depends on the true solution $f$, but never on the discretization parameters $\varepsilon$ and $\delta$.

Lemma 3.5 For all $t \in\left[0, T_{\varepsilon, \delta}\right], r>0$ and $n \in \mathcal{N}$,

$$
\bar{\rho}(t, r), \bar{p}(t, r),|\bar{\jmath}(t, r)|, \bar{m}(t, r),|\bar{\lambda}(t, r)|,|\bar{\mu}(t, r)|,|\bar{\lambda}(t, r)|,\left|\bar{\lambda}^{\prime}(t, r)\right|,\left|\bar{\mu}^{\prime}(t, r)\right| \leq C
$$

and

$$
\left|\dot{\bar{M}}_{n}(t)\right| \leq C \varepsilon^{3} .
$$

Moreover, for $r \notin[1 /(4 D), 3 D]$,

$$
\bar{\rho}(t, r)=\bar{p}(t, r)=\bar{\jmath}(t, r)=0 .
$$

Proof. Let $t \in\left[0, T_{\varepsilon, \delta}\right]$. By definition of $T_{\varepsilon, \delta}, r<1 /(4 D)$ implies $r-\bar{R}_{n}(t)<$ $1 /(4 D)-1 /(2 D) \leq-\delta$, and $r>3 D$ implies $r-\bar{R}_{n}(t)>3 D-2 D \geq \delta$. Thus

$$
\bar{\rho}(t, r)=0, r \notin[1 /(4 D), 3 D] .
$$

A bound on $\bar{\rho}$ of the desired type holds either by assumption or by definition of $T_{\varepsilon, \delta}$. It is obvious that $\bar{p},|\bar{\jmath}| \leq \bar{\rho}$ so that the assertions for $\bar{p}$ and $\bar{\jmath}$ follow. Using the bounds on the approximating source terms and (3.4) the remaining bounds follow from the definitions of the respective quantities and of $T_{\varepsilon, \delta}$.

Next we present an important tool for analyzing the approximating source terms: 
Lemma 3.6 Let $0<r_{0}<r_{1}$. For $t \in\left[0, T_{\varepsilon, \delta}\right]$ define

$$
I(t):=\left\{n \in \mathbb{N} \mid R_{n}(t) \in\left[r_{0}, r_{1}\right]\right\}, \bar{I}(t):=\left\{n \in \mathbb{N} \mid \bar{R}_{n}(t) \in\left[r_{0}, r_{1}\right]\right\} .
$$

Then

$$
\begin{aligned}
& |I(t)| \leq C \frac{r_{1}-r_{0}+\varepsilon}{\varepsilon^{3}}, \\
& |\bar{I}(t)| \leq C \frac{r_{1}-r_{0}+\|R(t)-\bar{R}(t)\|+\varepsilon}{\varepsilon^{3}},
\end{aligned}
$$

where $|I|$ denotes the number of elements of a set $I \subset \mathbb{N}$. Moreover,

$$
\sum_{n \in \bar{I}(t)} \bar{M}_{n}(t) \leq C\left(r_{1}-r_{0}+\delta\right) .
$$

Proof. The proof of the first assertion follows the one of [6, Lemma 2]. Fix $n \in I(t)$ and $(r, w, L) \in A_{n}$. Then Lemma 3.4 implies that

$$
R(t, 0, r, w, L) \mid \in\left[r_{0}-C \varepsilon, r_{1}+C \varepsilon\right],
$$

and thus

$$
\bigcup_{n \in I(t)} A_{n}(t) \subset\left[r_{0}-C \varepsilon, r_{1}+C \varepsilon\right] \times[-D, D] \times[0, D] .
$$

Using Lemma 2.1, the bound on $\lambda$ from Lemma 3.1, and the lower bound on meas $\left(A_{n}\right)$ we find

$$
\left(r_{1}-r_{0}+2 C \varepsilon\right) 2 D^{2} \geq \sum_{n \in I(t)} \operatorname{meas}\left(A_{n}(t)\right) \geq \frac{1}{C} \sum_{n \in I(t)} \operatorname{meas}\left(A_{n}\right) \geq C|I(t)| \varepsilon^{3},
$$

which implies the first assertion. The second one follows from the first, since

$$
\bar{I}(t) \subset\left\{n \in \mathcal{N} \mid R_{n}(t) \in\left[r_{0}-\|R(t)-\bar{R}(t)\|, r_{1}+\|R(t)-\bar{R}(t)\|\right]\right\} .
$$

To prove the third inequality define for $r \geq \delta / 2$,

$$
\bar{I}(t, r):=\left\{n \in \mathcal{N} \mid \bar{R}_{n}(t) \in[r-\delta / 2, r+\delta / 2]\right\} .
$$

Then $n \in \bar{I}(t, r)$ implies $\left|\bar{R}_{n}(t)-r\right| \leq \delta / 2$ and therefore $\chi_{\delta}\left(\bar{R}_{n}(t)-r\right) \geq 1 / 2$. Hence

$$
\begin{aligned}
\sum_{n \in \bar{I}(t, r)} \bar{M}_{n}(t) & \leq 2 \sum_{n \in \bar{I}(t, r)} \bar{M}_{n}(t) \chi_{\delta}\left(\bar{R}_{n}(t)-r\right) \\
& \leq 8 \pi r^{2} \delta \bar{\rho}(t, r) \leq 8 \pi r^{2} \delta \max \left\{C^{\star}, 2 D\right\}=C r^{2} \delta, r \geq \delta / 2 .
\end{aligned}
$$

Now define $k:=\left\lceil\left(r_{1}-r_{0}\right) / \delta\right\rceil$ and $s_{i}:=r_{0}+i \delta, i=0, \ldots, k$. Here $\lceil\zeta\rceil$ denotes the smallest integer larger than or equal to $\zeta$. Then $s_{0}=r_{0}$ and $s_{k} \geq r_{1}$, and therefore

$$
\bar{I}(t) \subset \bigcup_{i=0}^{k-1} \bar{I}\left(t, s_{i}+\delta / 2\right) .
$$


Using this inclusion and the previous estimate for every $i$ together with the fact that by definition of $T_{\varepsilon, \delta}$ only $r_{1} \leq 3 D$ needs to be considered we get the estimate

$$
\begin{aligned}
\sum_{n \in \bar{I}(t)} \bar{M}_{n}(t) & \leq \sum_{i=0}^{k-1} \sum_{n \in \bar{I}\left(t, s_{i}+\delta / 2\right)} \bar{M}_{n}(t) \leq \sum_{i=0}^{k-1} C\left(s_{i}+\delta / 2\right)^{2} \delta \\
& \leq C\left(r_{1}+\delta / 2\right)^{2} k \delta+\leq C\left\lceil\left(r_{1}-r_{0}\right) / \delta\right\rceil \delta \leq C\left(r_{1}-r_{0}+\delta\right),
\end{aligned}
$$

and the proof is complete.

In order to analyze the errors appearing at the source terms and the metric coefficients due to the discretization we define quantities that are intermediate between the true quantities and their approximations in the sense that the formulas for the latter source terms are evaluated at the true characteristics with the true weight functions. These intermediate quantities are denoted by double-bars:

$$
\overline{\bar{\rho}}(t, r):=\frac{1}{4 \pi r^{2} \delta} \sum_{n=1}^{N} E_{n}(t) M_{n}(t) \chi_{\delta}\left(r-R_{n}(t)\right)
$$

where analogously to (2.16),

$$
E_{n}(t):=\sqrt{1+W_{n}^{2}(t)+L_{n} / R_{n}^{2}(t)}
$$

with corresponding definitions for $\overline{\bar{p}}$ and $\overline{\bar{\jmath}}$. Moreover, $\overline{\bar{M}}_{n}$ is to be the solution of

$$
\dot{\bar{M}}_{n}(t)=-\left(\dot{\lambda}\left(t, R_{n}(t)\right)+\dot{R}_{n}(t) \lambda^{\prime}\left(t, R_{n}(t)\right)\right) \overline{\bar{M}}_{n}(t), \quad \overline{\bar{M}}_{n}(0)=M_{n}(0) .
$$

Note that we have, similar to $\bar{\rho}$ and $\bar{M},\|\overline{\bar{\rho}}(t)\|_{\infty}+\varepsilon^{-3} \overline{\bar{M}}(t) \leq C$ for all $t \in[0, T]$ and $\overline{\bar{\rho}}(t, r)=0$ for all $r \notin[1 /(4 D), 3 D], t \in[0, T]$.

Lemma 3.7 For all $t \in\left[0, T_{\varepsilon, \delta}\right]$,

$$
\begin{aligned}
\|\mu(t)-\bar{\mu}(t)\|_{\infty} & +\|\lambda(t)-\bar{\lambda}(t)\|_{\infty}+\|m(t)-\bar{m}(t)\|_{\infty} \\
\leq C\left(\|\rho(t)-\overline{\bar{\rho}}(t)\|_{\infty}+\|p(t)-\overline{\bar{p}}(t)\|_{\infty}\right. & \\
& \quad+\|R(t)-\bar{R}(t)\|+\|W(t)-\bar{W}(t)\|+\|M(t)-\bar{M}(t)\|) .
\end{aligned}
$$

Proof. Let $t \in\left[0, T_{\varepsilon, \delta}\right]$ and $r>0$. We start with analyzing the error $m-\bar{m}$. Due to the definition of $\bar{m}, \bar{\rho}$, and $\overline{\bar{\rho}}$,

$$
\begin{aligned}
& m(t, r)-\bar{m}(t, r)=4 \pi \int_{0}^{r}(\rho(t, s)-\bar{\rho}(t, s)) s^{2} d s=4 \pi \int_{0}^{r}(\rho(t, s)-\overline{\bar{\rho}}(t, s)) s^{2} d s \\
& \quad+\frac{1}{\delta} \int_{0}^{r} \sum_{n=1}^{N}\left(E_{n}(t) M_{n}(t) \chi_{\delta}\left(s-R_{n}(t)\right)-\bar{E}_{n}(t) \bar{M}_{n}(t) \chi_{\delta}\left(s-\bar{R}_{n}(t)\right)\right) d s \\
& =: F_{1}+F_{2} .
\end{aligned}
$$


Since for $r \geq 3 D$ we have $\rho(t, r)=\overline{\bar{\rho}}(t, r)=0$,

$$
\left|F_{1}\right| \leq C\|\rho(t)-\overline{\bar{\rho}}(t)\|_{\infty} .
$$

Using the definition of $\chi$, cf. Lemma 3.3, we have

$$
\begin{aligned}
\left|F_{2}\right| \leq & \left|\sum_{n=1}^{N}\left(M_{n}(t)-\bar{M}_{n}(t)\right) E_{n}(t) \chi\left(r-R_{n}(t)\right)\right| \\
& +\left|\sum_{n=1}^{N} \bar{M}_{n}(t)\left(E_{n}(t)-\bar{E}_{n}(t)\right) \chi\left(r-R_{n}(t)\right)\right| \\
& +\left|\sum_{n=1}^{N} \bar{M}_{n}(t) \bar{E}_{n}(t)\left(\chi\left(r-R_{n}(t)\right)-\chi\left(r-\bar{R}_{n}(t)\right)\right)\right|=: F_{21}+F_{22}+F_{23} .
\end{aligned}
$$

Since $E_{n}$ and $\chi$ are bounded and $\varepsilon^{3} N \leq C$ by (3.2),

$$
F_{21} \leq C \sum_{n=1}^{N}\left|M_{n}(t)-\bar{M}_{n}(t)\right| \leq C\|M(t)-\bar{M}(t)\| .
$$

Since $W_{n}(t), \bar{W}_{n}(t), 1 / R_{n}(t), 1 / \bar{R}_{n}(t)$, and $L_{n}$ are bounded,

$$
\left|E_{n}(t)-\bar{E}_{n}(t)\right| \leq C\left(\left|R_{n}(t)-\bar{R}_{n}(t)\right|+\left|W_{n}(t)-\bar{W}_{n}(t)\right|\right),
$$

and thus

$$
\begin{aligned}
F_{22} & \leq C \sum_{n=1}^{N} \bar{M}_{n}(t)(\|R(t)-\bar{R}(t)\|+\|W(t)-\bar{W}(t)\|) \\
& \leq C(\|R(t)-\bar{R}(t)\|+\|W(t)-\bar{W}(t)\|) .
\end{aligned}
$$

If we define

$$
I_{1}(t):=\left\{n \in \mathcal{N}|| R_{n}(t)-r \mid \leq\|R(t)-\bar{R}(t)\|_{\infty}+\delta\right\}
$$

then by Lemma 3.3 ,

$$
\chi\left(r-R_{n}(t)\right)=\chi\left(r-\bar{R}_{n}(t)\right), n \notin I_{1}(t),
$$

and by Lemma 3.6 ,

$$
\left|I_{1}(t)\right| \leq C \frac{\|R(t)-\bar{R}(t)\|_{\infty}+\delta}{\varepsilon^{3}}
$$

which implies, again with Lemma 3.3, that

$$
F_{23} \leq C \sum_{n \in I_{1}(t)} \bar{M}_{n}(t) \min \left\{\frac{\left|R_{n}(t)-\bar{R}_{n}(t)\right|}{\delta}, 1\right\} \leq C\|R(t)-\bar{R}(t)\| .
$$


This completes the desired estimate for $m-\bar{m}$. The estimate for $\lambda-\bar{\lambda}$ follows directly from the expressions for $\lambda$ and $\bar{\lambda}$ in terms of $m$ and $\bar{m}$, the fact that $\lambda(t, r)=\bar{\lambda}(t, r)=0$ for $r<1 /(4 D)$, and the estimate which we just completed.

It remains to consider $\mu-\bar{\mu}$. For $r<1 /(4 D)$ we have $\mu^{\prime}(t, r)=\bar{\mu}^{\prime}(t, r)=0$ and therefore $|\mu(t, r)-\bar{\mu}(t, r)|=|\mu(t, 1 /(4 D))-\bar{\mu}(t, 1 /(4 D))|$. Let $r \geq 1 /(4 D)$. Then the definitions of $\mu, \bar{\mu}, \mu^{\prime}$, and $\bar{\mu}^{\prime}$ yield

$$
\begin{aligned}
|\mu(t, r)-\bar{\mu}(t, r)|= & \left|\int_{r}^{\infty} \bar{\mu}^{\prime}(t, s)-\mu^{\prime}(t, s) d s\right| \\
\leq & \int_{r}^{\infty} e^{2 \bar{\lambda}(t, s)}\left|\frac{\bar{m}(t, s)}{s^{2}}-\frac{m(t, s)}{s^{2}}\right| d s \\
& +\int_{r}^{\infty}\left|e^{2 \bar{\lambda}(t, s)}-e^{2 \lambda(t, s)}\right|\left(\frac{m(t, s)}{s^{2}}+4 \pi s p(t, s)\right) d s \\
& +\left|\int_{r}^{\infty} 4 \pi s e^{2 \bar{\lambda}(t, s)}(\bar{p}(t, s)-p(t, s)) d s\right| \\
\leq & C\|m(t)-\bar{m}(t)\|_{\infty}+C\|\lambda(t)-\bar{\lambda}(t)\|_{\infty} \\
& +\left|\int_{r}^{\infty} 4 \pi s e^{2 \bar{\lambda}(t, s)}(\bar{p}(t, s)-p(t, s)) d s\right|
\end{aligned}
$$

We split the difference $\bar{p}-p$ into $\bar{p}-\overline{\bar{p}}$ and $\overline{\bar{p}}-p$. The integral resulting from the second term can obviously be estimated as desired. As to the first term,

$$
\begin{aligned}
\mid \int_{r}^{\infty} 4 & \pi s e^{2 \bar{\lambda}(t, s)}(\overline{\bar{p}}(t, s)-\bar{p}(t, s)) d s \mid \\
\leq & \frac{C}{\delta} \sum_{n=1}^{N}\left|M_{n}(t)-\bar{M}_{n}(t)\right| \int_{1 /(4 D)}^{3 D} \chi_{\delta}\left(s-R_{n}(t)\right) d s \\
& +\frac{C}{\delta} \sum_{n=1}^{N} \bar{M}_{n}(t)\left|\frac{W_{n}^{2}(t)}{E_{n}(t)}-\frac{\bar{W}_{n}^{2}(t)}{\bar{E}_{n}(t)}\right| \int_{1 /(4 D)}^{3 D} \chi_{\delta}\left(s-R_{n}(t)\right) d s \\
& +\frac{C}{\delta} \sum_{n=1}^{N} \bar{M}_{n}(t)\left|\int_{r}^{\infty} \frac{e^{2 \bar{\lambda}(t, s)}}{s}\left(\chi_{\delta}\left(s-R_{n}(t)\right)-\chi_{\delta}\left(s-\bar{R}_{n}(t)\right)\right) d s\right| \\
= & : F_{3}+F_{4}+F_{5}
\end{aligned}
$$

where we used the bounds on the supports of $\overline{\bar{p}}$ and $\bar{p}$ and recalled that $r \geq 1 /(4 D)$. The first two terms are comparatively easy to deal with: Define $I_{2}(t, s):=\left\{n \in \mathcal{N}|| s-R_{n}(t) \mid \leq \delta\right\}$. By Lemma 3.6, $\left|I_{2}(t, s)\right| \leq C(\delta+\varepsilon) \varepsilon^{-3} \leq$ $C \delta \varepsilon^{-3}$ for all $s \geq r$. Thus

$$
\begin{aligned}
F_{3} & \leq \frac{C}{\delta} \varepsilon^{3}\|M(t)-\bar{M}(t)\| \int_{1 /(4 D)}^{3 D}\left|I_{2}(t, s)\right| d s \leq C\|M(t)-\bar{M}(t)\|, \\
F_{4} & \leq \frac{C}{\delta} \varepsilon^{3}(\|R(t)-\bar{R}(t)\|+\|W(t)-\bar{W}(t)\|) \int_{1 /(4 D)}^{3 D}\left|I_{2}(t, s)\right| d s \\
& \leq C(\|R(t)-\bar{R}(t)\|+\|W(t)-\bar{W}(t)\|) .
\end{aligned}
$$


To deal with $F_{5}$ we fix some $n \in \mathcal{N}$ and assume without loss of generality that $\bar{R}_{n}(t) \leq R_{n}(t)$. A change of variables yields

$$
\begin{aligned}
\mid \int_{r}^{\infty} & \frac{e^{2 \bar{\lambda}(t, s)}}{s}\left(\chi_{\delta}\left(s-R_{n}(t)\right)-\chi_{\delta}\left(s-\bar{R}_{n}(t)\right)\right) d s \mid \\
= & \left|\int_{r-R_{n}(t)}^{\infty} \frac{e^{2 \bar{\lambda}\left(t, z+R_{n}(t)\right)}}{z+R_{n}(t)} \chi_{\delta}(z) d z-\int_{r-\bar{R}_{n}(t)}^{\infty} \frac{e^{2 \bar{\lambda}\left(t, z+\bar{R}_{n}(t)\right)}}{z+\bar{R}_{n}(t)} \chi_{\delta}(z) d z\right| \\
\leq & \int_{r-R_{n}(t)}^{r-\bar{R}_{n}(t)} \frac{e^{2 \bar{\lambda}\left(t, z+R_{n}(t)\right)}}{z+R_{n}(t)} \chi_{\delta}(z) d z \\
& +\int_{r-\bar{R}_{n}(t)}^{\infty}\left|\frac{e^{2 \bar{\lambda}\left(t, z+R_{n}(t)\right)}}{z+R_{n}(t)}-\frac{e^{2 \bar{\lambda}\left(t, z+\bar{R}_{n}(t)\right)}}{z+\bar{R}_{n}(t)}\right| \chi_{\delta}(z) d z \\
\leq & C \int_{r-R_{n}(t)}^{r-\bar{R}_{n}(t)} \chi_{\delta}(z) d z+C \int_{r-\bar{R}_{n}(t)}^{\infty} \chi_{\delta}(z) d z\left|R_{n}(t)-\bar{R}_{n}(t)\right| \\
\leq & C \min \left\{\left|R_{n}(t)-\bar{R}_{n}(t)\right|, \delta\right\}+C \delta\|R(t)-\bar{R}(t)\| .
\end{aligned}
$$

Define

$$
\begin{aligned}
I_{3}(t): & =\left\{n \in \mathcal{N} \mid\left[r-R_{n}(t), r-\bar{R}_{n}(t)\right] \cap[-\delta,+\delta] \neq \emptyset\right\} \\
& \subset\left\{n \in \mathcal{N}|| R_{n}(t)-r \mid \leq \delta+\|R(t)-\bar{R}(t)\|\right\} .
\end{aligned}
$$

Then Lemma 3.6 yields $\left|I_{3}(t)\right| \leq C \varepsilon^{-3}(\delta+\|R(t)-\bar{R}(t)\|)$, and putting everything together, we obtain

$$
\begin{aligned}
\left|F_{5}\right| & \leq \frac{C \varepsilon^{3}}{\delta} \sum_{n \in I_{3}(t)} \min \left\{\left|R_{n}(t)-\bar{R}_{n}(t)\right|, \delta\right\}+\frac{C \varepsilon^{3}}{\delta} \sum_{n=1}^{N} \delta\|R(t)-\bar{R}(t)\| \\
& \leq C\|R(t)-\bar{R}(t)\|
\end{aligned}
$$

by (3.2), and the proof is complete.

Next we estimate the differences of the intermediate, double-barred source terms and their true counterparts:

Lemma 3.8 For all $t \in\left[0, T_{\varepsilon, \delta}\right]$,

$$
\begin{aligned}
& \|\rho(t)-\overline{\bar{\rho}}(t)\|_{\infty} \leq C(\delta+\varepsilon / \delta), \\
& \|\rho(t)-\overline{\bar{\rho}}(t)\|_{\infty} \leq C\left(\delta^{2}+\varepsilon / \delta\right), \text { provided } \rho^{\prime \prime} \in C\left([0, T] \times \mathbb{R}^{+}\right),
\end{aligned}
$$

and the analogous assertions hold for $p$ and $\mathrm{J}$.

Proof. We restrict ourselves to considering $\rho ; p$ and $\jmath$ can be dealt with in the same fashion. Let $t \in\left[0, T_{\varepsilon, \delta}\right]$ and $r>0$. Then

$$
4 \pi r^{2} \delta \rho(t, r)=\int_{r-\delta}^{r+\delta} 4 \pi s^{2} \rho(t, s) \chi_{\delta}(r-s) d s
$$




$$
\begin{aligned}
& +\int_{r-\delta}^{r+\delta} 4 \pi\left(r^{2} \rho(t, r)-s^{2} \rho(t, s)\right) \chi_{\delta}(r-s) d s \\
= & F_{1}+F_{2}, \\
4 \pi r^{2} \delta \overline{\bar{\rho}}(t, r)= & \sum_{n=1}^{N} E_{n}(t) \chi_{\delta}\left(r-R_{n}(t)\right) \int_{A_{n}(t)} f(t, z) d z \\
= & \sum_{n=1}^{N} \int_{A_{n}(t)} E f(t, z) \chi_{\delta}(r-|x|) d z \\
& +\sum_{n=1}^{N} \int_{A_{n}(t)}\left(E_{n}(t)-E\right) f(t, z) \chi_{\delta}\left(r-R_{n}(t)\right) d z \\
& +\sum_{n=1}^{N} \int_{A_{n}(t)} E f(t, z)\left(\chi_{\delta}\left(r-R_{n}(t)\right)-\chi_{\delta}(r-|x|)\right) d z \\
=: & F_{3}+F_{4}+F_{5} .
\end{aligned}
$$

Clearly, $F_{1}=F_{3}$. Since $\rho(t, r)=\overline{\bar{\rho}}(t, r)=0$ for all $r \notin[1 /(4 D), 3 D]$,

$$
|\rho(t, r)-\overline{\bar{\rho}}(t, r)| \leq \frac{C}{\delta}\left(\left|F_{2}\right|+\left|F_{4}\right|+\left|F_{5}\right|\right) .
$$

Using the fact that we assume a bound on the derivative of $s^{2} \rho(t, s)$ with respect to $s$ we find

$$
\left|F_{2}\right| \leq C \int_{r-\delta}^{r+\delta}|r-s| \chi_{\delta}(r-s) d s \leq C \delta^{2} .
$$

To estimate $F_{4}$ let $z=(x, v) \in A_{n}(t)$. Let $Z_{n}(t)=\left(X_{n}(t), V_{n}(t)\right)$ be any Cartesian characteristic starting at a point with $(r, w, L)$ coordinates $\left(R_{n}(0), W_{n}(0), L_{n}\right)$. Then $Z_{n}(t) \in A_{n}(t)$ and $\left|z-Z_{n}(t)\right| \leq \operatorname{diam}\left(A_{n}(t)\right) \leq C \varepsilon$ by Lemma 3.4. The function $E$ used to compute $\rho$ and $\overline{\bar{\rho}}$ is Lipschitz on the relevant domain; the same is true for $p$ and $\jmath$. If we define $I_{1}(t):=\left\{n \in \mathcal{N}|| R_{n}(t)-r \mid \leq \delta\right\}$ then Lemma 3.6 yields $\left|I_{1}(t)\right| \leq C \delta \varepsilon^{-3}$ and therefore,

$$
\begin{aligned}
\left|F_{4}\right| & \leq \sum_{n \in I_{1}(t)} \int_{A_{n}(t)}\left|E_{n}(t)-E\right| f(t, z) d z \\
& \leq \sum_{n \in I_{1}(t)} C \operatorname{diam}\left(A_{n}(t)\right) \int_{A_{n}(t)} f(t, z) d z \leq C \delta \varepsilon .
\end{aligned}
$$

For $F_{5}$ we define

$$
I_{2}(t):=\left\{n \in \mathcal{N}|| R_{n}(t)-r \mid \leq \delta+\max _{m \in \mathcal{N}} \operatorname{diam}\left(A_{m}(t)\right)\right\} .
$$

Then $\chi_{\delta}\left(r-R_{n}(t)\right)=\chi_{\delta}(r-|x|)$ for all $n \notin I_{2}(t)$ and $z \in A_{n}(t)$, and by Lemma 3.6, $\left|I_{2}(t)\right| \leq C \varepsilon^{-3}\left(\delta+\max _{m \in \mathcal{N}} \operatorname{diam}\left(A_{m}(t)\right)+\varepsilon\right) \leq C \varepsilon^{-3} \delta$. Hence

$$
\left|F_{5}\right| \leq \frac{1}{\delta} \sum_{n \in I_{2}(t)} \int_{A_{n}(t)} E f(t, z)\left|R_{n}(t)-\right| x|| d z
$$




$$
\leq \frac{C}{\delta} \sum_{n \in I_{2}(t)} \operatorname{diam}\left(A_{n}(t)\right) M_{n}(t) \leq C \varepsilon,
$$

and the first assertion is proven.

To prove the second one, we have to sharpen the estimate for $F_{2}$ in the case that $\rho^{\prime \prime}$ exists and is continuous and thus bounded on the relevant domain. First we rewrite this term; since throughout the argument $t$ remains fixed we suppress this variable:

$$
\begin{aligned}
F_{2}= & 4 \pi \int_{r-\delta}^{r+\delta}\left(r^{2}-s^{2}\right) \rho(r) \chi_{\delta}(r-s) d s \\
& +4 \pi \int_{r-\delta}^{r+\delta}\left(\rho(r)-\frac{1}{2}(\rho(r+\delta)+\rho(r-\delta))\right) s^{2} \chi_{\delta}(r-s) d s \\
& +4 \pi \int_{r-\delta}^{r+\delta}\left(\frac{1}{2}(\rho(r+\delta)+\rho(r-\delta))-\rho(s)\right) s^{2} \chi_{\delta}(r-s) d s \\
= & F_{6}+F_{7}+F_{8} .
\end{aligned}
$$

By the mean value theorem

$$
\left|F_{6}\right| \leq C\left|\int_{r-\delta}^{r+\delta}\left(r^{2}-s^{2}\right) d s\right|=C \delta^{3} .
$$

To analyze $F_{7}$ we use the extra regularity of $\rho$. By Taylor expansion,

$$
\left|F_{7}\right| \leq C \int_{r-\delta}^{r+\delta}\left|\rho(r)-\frac{1}{2}(\rho(r+\delta)+\rho(r-\delta))\right| s^{2} d s \leq C \delta^{3} .
$$

Finally, by the mean value theorem and Taylor expansion,

$$
\begin{aligned}
\left|F_{8}\right| \leq C\left|\int_{r-\delta}^{r+\delta}\left(\frac{1}{2}(\rho(r+\delta)+\rho(r-\delta))-\rho(s)\right) d s\right| \\
=C\left|\delta \rho(r+\delta)+\delta \rho(r-\delta)-\int_{r}^{r+\delta} \rho(s) d s-\int_{r-\delta}^{r} \rho(s) d s\right| \\
\leq C \mid \delta \rho(r+\delta)+\delta \rho(r-\delta)-\int_{r}^{r+\delta}\left(\rho(r+\delta)+\rho^{\prime}(r+\delta)(s-r-\delta)\right) d s \\
\quad-\int_{r-\delta}^{r}\left(\rho(r-\delta)+\rho^{\prime}(r-\delta)(s-r+\delta)\right) d s \mid \\
\quad+C \int_{r}^{r+\delta}(s-r-\delta)^{2} d s+C \int_{r-\delta}^{r}(s-r+\delta)^{2} d s \\
=C\left|\frac{1}{2} \delta^{2} \rho^{\prime}(r+\delta)-\frac{1}{2} \delta^{2} \rho^{\prime}(r-\delta)\right|+C \delta^{3} \\
\leq C \delta^{3},
\end{aligned}
$$


and the proof is complete.

The next lemma will complement the previous one in that we now estimate the differences between the approximations of the source terms and the corresponding intermediate double-barred quantities. It is at this point that we loose one order of $\delta$ in the error estimates for the sources compared to the other quantities in Theorem 2.2:

Lemma 3.9 For all $t \in\left[0, T_{\varepsilon, \delta}\right]$,

$$
\|\bar{\rho}(t)-\overline{\bar{\rho}}(t)\|_{\infty} \leq C\left(\frac{\|R(t)-\bar{R}(t)\|}{\delta}+\|W(t)-\bar{W}(t)\|+\|M(t)-\bar{M}(t)\|\right),
$$

in particular, $\|\bar{\rho}(0)-\overline{\bar{\rho}}(0)\|_{\infty}=0$. The same estimates hold for $p$ and $\mathrm{J}$.

Proof. Since $\bar{\rho}(t, r)=\overline{\bar{\rho}}(t, r)=0$ for $r \notin[1 /(4 D), 3 D]$ we only need to consider $r \in[1 /(4 D), 3 D]$. By definition

$$
\begin{aligned}
\bar{\rho}(t, r)-\overline{\bar{\rho}}(t, r)= & \frac{1}{4 \pi r^{2} \delta} \sum_{n=1}^{N} \bar{E}_{n}(t) \bar{M}_{n}(t)\left(\chi_{\delta}\left(r-\bar{R}_{n}(t)\right)-\chi_{\delta}\left(r-R_{n}(t)\right)\right) \\
& +\frac{1}{4 \pi r^{2} \delta} \sum_{n=1}^{N}\left(\bar{E}_{n}(t) \bar{M}_{n}(t)-E_{n}(t) M_{n}(t)\right) \chi_{\delta}\left(r-R_{n}(t)\right) .
\end{aligned}
$$

Let $I_{1}(t):=\left\{n \in \mathcal{N}|| R_{n}(t)-r \mid \leq \delta\right\}$ and $I_{2}(t):=\left\{n \in \mathcal{N}|| \bar{R}_{n}(t)-r \mid \leq \delta\right\}$. Then Lemma 3.6 implies that $\left|I_{1}(t)\right| \leq C \varepsilon^{-3} \delta$ and $\sum_{n \in I_{2}(t)} \bar{M}_{n}(t) \leq C \delta$. Moreover, $n \notin$ $I_{1}(t) \cup I_{2}(t)$ implies $\chi_{\delta}\left(r-\bar{R}_{n}(t)\right)=0=\chi_{\delta}\left(r-R_{n}(t)\right)$. Using similar estimates as in the lemmas above we find

$$
\begin{aligned}
& |\bar{\rho}(t, r)-\overline{\bar{\rho}}(t, r)| \leq \frac{C}{\delta} \sum_{n \in I_{1}(t) \cup I_{2}(t)} \bar{M}_{n}(t)\left|R_{n}(t)-\bar{R}_{n}(t)\right| / \delta \\
& \quad+\frac{C \varepsilon^{3}}{\delta} \sum_{n \in I_{1}(t)}\left(\left|R_{n}(t)-\bar{R}_{n}(t)\right|+\left|W_{n}(t)-\bar{W}_{n}(t)\right|+\varepsilon^{-3}\left|M_{n}(t)-\bar{M}_{n}(t)\right|\right) \\
& \leq C \frac{\|R(t)-\bar{R}(t)\|}{\delta^{2}}\left(\sum_{n \in I_{1}(t)} \varepsilon^{3}+\sum_{n \in I_{2}(t)} \bar{M}_{n}(t)\right) \\
& \quad+\frac{C \varepsilon^{3}}{\delta}\left|I_{1}(t)\right|(\|R(t)-\bar{R}(t)\|+\|W(t)-\bar{W}(t)\|+\|M(t)-\bar{M}(t)\|),
\end{aligned}
$$

and the proof is complete.

We now turn to the analysis of the evolution equations.

Lemma 3.10 For all $t \in\left[0, T_{\varepsilon, \delta}\right]$,

$$
\|M(t)-\overline{\bar{M}}(t)\|:=\varepsilon^{-3} \max _{n \in \mathcal{N}} \sup _{s \in[0, t]}\left|M_{n}(s)-\overline{\bar{M}}_{n}(s)\right| \leq C \varepsilon .
$$


Proof. Let $t \in\left[0, T_{\varepsilon, \delta}\right]$ and $n \in \mathcal{N}$. The definition of $\overline{\bar{M}}_{n}$ and Lemma 2.1 with $A=A_{n}$ yields

$$
\begin{aligned}
M_{n}(t)-\overline{\bar{M}}_{n}(t)= & \int_{0}^{t}\left(\dot{\lambda}\left(s, R_{n}(s)\right)+\dot{R}_{n}(s) \lambda^{\prime}\left(s, R_{n}(s)\right)\right) \overline{\bar{M}}_{n}(s) d s \\
& -\int_{0}^{t} \int_{A_{n}(s)} f(s, z)\left(\dot{\lambda}(s,|x|)+\dot{R}(s, 0, Z(0, s, z)) \lambda^{\prime}(s,|x|)\right) d z d s \\
= & \int_{0}^{t}\left[\left(\dot{\lambda}\left(s, R_{n}(s)\right)+\dot{R}_{n}(s) \lambda^{\prime}\left(s, R_{n}(s)\right)\right)\left(\overline{\bar{M}}_{n}(s)-M_{n}(s)\right)\right] d s \\
& +\int_{0}^{t} \int_{A_{n}(s)} f(s, z)\left[\dot{\lambda}\left(s, R_{n}(s)\right)-\dot{\lambda}(s,|x|)+\dot{R}_{n}(s) \lambda^{\prime}\left(s, R_{n}(s)\right)\right. \\
& \left.-\dot{R}(s, 0, Z(0, s, z)) \lambda^{\prime}(s,|x|)\right] d z d s .
\end{aligned}
$$

For $z=(x, v) \in A_{n}(s)$,

$$
\left|\dot{\lambda}\left(s, R_{n}(s)\right)-\dot{\lambda}(s,|x|)\right| \leq C\left|R_{n}(s)-\right| x|| \leq C \operatorname{diam}\left(A_{n}(s)\right) \leq C \varepsilon,
$$

and

$$
\begin{aligned}
\mid \dot{R}_{n}(s) \lambda^{\prime}\left(s, R_{n}(s)\right)- & \dot{R}(s, 0, Z(0, s, z)) \lambda^{\prime}(s,|x|) \mid \\
\leq & \left|\dot{R}\left(s, 0, z_{n}\right)-\dot{R}(s, 0, Z(0, s, z))\right|\left|\lambda^{\prime}\left(s, R_{n}(s)\right)\right| \\
& +|\dot{R}(s, 0, Z(0, s, z))|\left|\lambda^{\prime}\left(s, R_{n}(s)\right)-\lambda^{\prime}(s,|x|)\right| \\
\leq & C\left(\left|z_{n}-Z(0, s, z)\right|+\left|R_{n}(s)-\right| x||\right) \\
\leq & C\left(\operatorname{diam}\left(A_{n}(0)\right)+\operatorname{diam}\left(A_{n}(s)\right)\right) \leq C \varepsilon
\end{aligned}
$$

Hence,

$$
\begin{aligned}
\left|M_{n}(t)-\overline{\bar{M}}_{n}(t)\right| & \leq C \int_{0}^{t}\left|M_{n}(s)-\overline{\bar{M}}_{n}(s)\right| d s+C \varepsilon \int_{0}^{t} M_{n}(s) d s \\
& \leq C \int_{0}^{t}\left|M_{n}(s)-\overline{\bar{M}}_{n}(s)\right| d s+C \varepsilon^{4}
\end{aligned}
$$

and a Gronwall argument completes the proof.

Lemma 3.11 For all $t \in\left[0, T_{\varepsilon, \delta}\right]$ and $n \in \mathcal{N}$,

$$
\begin{aligned}
\left|\dot{R}_{n}(t)-\dot{\bar{R}}_{n}(t)\right| \leq C(\mid & R_{n}(t)-\bar{R}_{n}(t)|+| W_{n}(t)-\bar{W}_{n}(t) \mid \\
+ & \left.\|\mu(t)-\bar{\mu}(t)\|_{\infty}+\|\lambda(t)-\bar{\lambda}(t)\|_{\infty}\right) .
\end{aligned}
$$

Proof. By the equations for $\dot{R}_{n}(t)$ and $\dot{\bar{R}}_{n}(t)$ and straight forward estimates,

$$
\begin{aligned}
\left|\dot{R}_{n}(t)-\dot{\bar{R}}_{n}(t)\right| \leq & \left|e^{(\mu-\lambda)\left(t, R_{n}(t)\right)}-e^{(\mu-\lambda)\left(t, \bar{R}_{n}(t)\right)}\right| \\
& +\left|e^{(\mu-\lambda)\left(t, \bar{R}_{n}(t)\right)}-e^{(\bar{\mu}-\bar{\lambda})\left(t, \bar{R}_{n}(t)\right)}\right|+\left|\frac{W_{n}(t)}{E_{n}(t)}-\frac{\bar{W}_{n}(t)}{\bar{E}_{n}(t)}\right|,
\end{aligned}
$$


and the assertion follows.

The use of the following technical lemma will become obvious later:

Lemma 3.12 Let $g_{n, m} \in C^{1}\left(\left[0, T_{\varepsilon, \delta}\right]\right), m, n \in \mathcal{N}$. Then for all $t \in\left[0, T_{\varepsilon, \delta}\right]$ and $m \in \mathcal{N}$

$$
\begin{aligned}
& \mid \frac{1}{\delta} \sum_{n=1}^{N} \int_{0}^{t} g_{n, m}(s)\left[\chi_{\delta}\left(\bar{R}_{m}(s)-R_{n}(s)\right)\left(\dot{\bar{R}}_{m}(s)-\dot{R}_{n}(s)\right)\right. \\
& \left.-\chi_{\delta}\left(\bar{R}_{m}(s)-\bar{R}_{n}(s)\right)\left(\dot{\bar{R}}_{m}(s)-\dot{\bar{R}}_{n}(s)\right)\right] d s \mid \\
& \leq C \varepsilon^{-3} \max _{k, l \in \mathcal{N}}\left(\left\|g_{k, l}\right\|_{\infty}+\left\|\dot{g}_{k, l}\right\|_{\infty}\right)\|R(t)-\bar{R}(t)\| .
\end{aligned}
$$

(Note that $C$ does not depend on the functions $g_{m, n}$.)

Proof. Let $m \in \mathcal{N}$ be fixed, denote the left hand side of the inequality by $S$, and define

$$
d_{n}(s):=\bar{R}_{m}(s)-R_{n}(s), \bar{d}_{n}(s):=\bar{R}_{m}(s)-\bar{R}_{n}(s), s \in\left[0, T_{\varepsilon, \delta}\right] .
$$

Integration by parts and $d_{n}(0)=\bar{d}_{n}(0)$ implies that

$$
S=\sum_{n=1}^{N}\left[g_{n, m}(t)\left(\chi\left(d_{n}(t)\right)-\chi\left(\bar{d}_{n}(t)\right)\right)-\int_{0}^{t} \dot{g}_{n, m}(s)\left(\chi\left(d_{n}(s)\right)-\chi\left(\bar{d}_{n}(s)\right)\right) d s\right] .
$$

For $s \in\left[0, T_{\varepsilon, \delta}\right]$ define

$$
I(s):=\left\{n \in \mathcal{N}|| R_{n}(s)-\bar{R}_{m}(s) \mid \leq \delta+\|R(s)-\bar{R}(s)\|\right\} .
$$

By Lemma 3.6,

$$
|I(s)| \leq C \varepsilon^{-3}(\delta+\|R(s)-\bar{R}(s)\|+\varepsilon) \leq C \varepsilon^{-3}(\delta+\|R(t)-\bar{R}(t)\|), s \in\left[0, T_{\varepsilon, \delta}\right] .
$$

If $R_{n}(s)<\bar{R}_{m}(s)-\delta-\|R(s)-\bar{R}(s)\|$ then $d_{n}(s) \geq \delta$ and $\bar{d}_{n}(s) \geq \delta$, and hence $\chi\left(d_{n}(s)\right)=1=\chi\left(\bar{d}_{n}(s)\right)$. If $R_{n}(s)>\bar{R}_{m}(s)+\delta+\|R(s)-\bar{R}(s)\|$ then $d_{n}(s) \leq-\delta$ and $\bar{d}_{n}(s) \leq-\delta$, and hence $\chi\left(d_{n}(s)\right)=0=\chi\left(\bar{d}_{n}(s)\right)$. Hence, using Lemma 3.3 ,

$$
\begin{aligned}
|S| \leq & \sum_{n \in I(t)}\left|g_{n, m}(t)\right|\left|\chi\left(d_{n}(t)\right)-\chi\left(\bar{d}_{n}(t)\right)\right| \\
& +\int_{0}^{t} \max _{k, l \in \mathcal{N}}\left|\dot{g}_{k, l}(s)\right| \sum_{n \in I(s)}\left|\chi\left(d_{n}(s)\right)-\chi\left(\bar{d}_{n}(s)\right)\right| d s \\
\leq & \max _{k, l \in \mathcal{N}}\left\|g_{k, l}\right\|_{\infty}|I(t)| \min \{\|R(t)-\bar{R}(t)\| / \delta, 1\} \\
& +\max _{k, l \in \mathcal{N}}\left\|\dot{g}_{k, l}\right\|_{\infty} \int_{0}^{t}|I(s)| \min \{\|R(s)-\bar{R}(s)\| / \delta, 1\} d s \\
\leq & C \max _{k, l \in \mathcal{N}}\left(\left\|g_{k, l}\right\|_{\infty}+\left\|\dot{g}_{k, l}\right\|_{\infty}\right)(\delta+\|R(t)-\bar{R}(t)\|) \varepsilon^{-3} \\
& \min \{\|R(t)-\bar{R}(t)\| / \delta, 1\},
\end{aligned}
$$

and the assertion follows. 
Lemma 3.13 Let

$$
\begin{aligned}
e(s):= & \|\rho(s)-\overline{\bar{\rho}}(s)\|_{\infty}+\|p(s)-\overline{\bar{p}}(s)\|_{\infty}+\|\jmath(s)-\overline{\bar{\jmath}}(s)\|_{\infty} \\
& +\|W(s)-\bar{W}(s)\|+\|M(s)-\bar{M}(s)\| .
\end{aligned}
$$

Then

$$
\|\overline{\bar{M}}(t)-\bar{M}(t)\| \leq C \int_{0}^{t} e(s) d s+C\|R(t)-\bar{R}(t)\|, t \in\left[0, T_{\varepsilon, \delta}\right] .
$$

Proof. Let $t \in\left[0, T_{\varepsilon, \delta}\right]$ and $m \in \mathcal{N}$. By the definitions of $\bar{M}_{m}$ and $\overline{\bar{M}}_{m}$,

$$
\begin{aligned}
\overline{\bar{M}}_{m}(t) & -\bar{M}_{m}(t) \\
= & \int_{0}^{t}\left[\overline{\dot{\lambda}}\left(s, \bar{R}_{m}(s)\right)+\dot{\bar{R}}_{m}(s) \bar{\lambda}^{\prime}\left(s, \bar{R}_{m}(s)\right)\right] \bar{M}_{m}(s) d s \\
& \quad \int_{0}^{t}\left[\dot{\lambda}\left(s, R_{m}(s)\right)+\dot{R}_{m}(s) \lambda^{\prime}\left(s, R_{m}(s)\right)\right] \bar{M}_{m}(s) d s \\
= & \int_{0}^{t}\left[\overline{\dot{\lambda}}\left(s, \bar{R}_{m}(s)\right)+\dot{\bar{R}}_{m}(s) \bar{\lambda}^{\prime}\left(s, \bar{R}_{m}(s)\right)\right. \\
& \left.\quad-\dot{\lambda}\left(s, \bar{R}_{m}(s)\right)-\dot{\bar{R}}_{m}(s) \lambda^{\prime}\left(s, \bar{R}_{m}(s)\right)\right] \bar{M}_{m}(s) d s \\
& +\int_{0}^{t}\left[\dot{\lambda}\left(s, \bar{R}_{m}(s)\right)-\dot{\lambda}_{\left(s, R_{m}\right.}(s)\right)+\left(\dot{\bar{R}}_{m}(s)-\dot{R}_{m}(s)\right) \lambda^{\prime}\left(s, \bar{R}_{m}(s)\right) \\
& \left.\quad+\dot{R}_{m}(s)\left(\lambda^{\prime}\left(s, \bar{R}_{m}(s)\right)-\lambda^{\prime}\left(s, R_{m}(s)\right)\right)\right] \bar{M}_{m}(s) d s \\
& +\int_{0}^{t}\left[\dot{\lambda}\left(s, R_{m}(s)\right)+\dot{R}_{m}(s) \lambda^{\prime}\left(s, R_{m}(s)\right)\right]\left(\bar{M}_{m}(s)-\overline{\bar{M}}_{m}(s)\right) d s \\
= & : F_{1}+F_{2}+F_{3} .
\end{aligned}
$$

By Lemma 3.1,

$$
\left|F_{3}\right| \leq C \int_{0}^{t}\left|\bar{M}_{m}(s)-\overline{\bar{M}}_{m}(s)\right| d s .
$$

By Lemma 3.1 and Lemma 3.11,

$$
\begin{gathered}
\left|F_{2}\right| \leq C \varepsilon^{3} \int_{0}^{t}\left(\left|R_{m}(s)-\bar{R}_{m}(s)\right|+\left|\dot{R}_{m}(s)-\dot{\bar{R}}_{m}(s)\right|\right) d s \\
\leq C \varepsilon^{3} \int_{0}^{t}\left(\|\mu(s)-\bar{\mu}(s)\|_{\infty}+\|\lambda(s)-\bar{\lambda}(s)\|_{\infty}\right. \\
\quad+\|R(s)-\bar{R}(s)\|+\|W(s)-\bar{W}(s)\|) d s .
\end{gathered}
$$

Now we use the formulas for the derivatives of $\lambda, \bar{\lambda}$ respectively where we smuggle in the intermediate double-barred source terms to estimate $F_{1}$ :

$\left|F_{1}\right|=\mid 4 \pi \int_{0}^{t} \bar{R}_{m}(s) \bar{M}_{m}(s)$ 


$$
\begin{aligned}
& {\left[e^{(\lambda+\mu)\left(s, \bar{R}_{m}(s)\right)} \jmath\left(s, \bar{R}_{m}(s)\right)-e^{(\bar{\lambda}+\bar{\mu})\left(s, \bar{R}_{m}(s)\right)} \bar{\jmath}\left(s, \bar{R}_{m}(s)\right)\right.} \\
& +\dot{\bar{R}}_{m}(s)\left(e^{2 \bar{\lambda}\left(s, \bar{R}_{m}(s)\right)}\left(\bar{\rho}\left(s, \bar{R}_{m}(s)\right)-\frac{\bar{m}\left(s, \bar{R}_{m}(s)\right)}{\bar{R}_{m}^{3}(s)}\right)\right. \\
& \left.\left.-e^{2 \lambda\left(s, \bar{R}_{m}(s)\right)}\left(\rho\left(s, \bar{R}_{m}(s)\right)-\frac{m\left(s, \bar{R}_{m}(s)\right)}{\bar{R}_{m}^{3}(s)}\right)\right)\right] d s \mid \\
& \leq C \varepsilon^{-3} \int_{0}^{t}\left|e^{2 \bar{\lambda}\left(s, \bar{R}_{m}(s)\right)} \frac{\bar{m}\left(s, \bar{R}_{m}(s)\right)}{\bar{R}_{m}^{3}(s)}-e^{2 \lambda\left(s, \bar{R}_{m}(s)\right)} \frac{m\left(s, \bar{R}_{m}(s)\right)}{\bar{R}_{m}^{3}(s)}\right| d s \\
& +\mid 4 \pi \int_{0}^{t} \bar{R}_{m}(s) \bar{M}_{m}(s)\left[\left(e^{(\lambda+\mu)\left(s, \bar{R}_{m}(s)\right)}-e^{(\bar{\lambda}+\bar{\mu})\left(s, \bar{R}_{m}(s)\right)}\right) \jmath\left(s, \bar{R}_{m}(s)\right)\right. \\
& \left.-\dot{\bar{R}}_{m}(s)\left(e^{2 \lambda\left(s, \bar{R}_{m}(s)\right)}-e^{2 \bar{\lambda}\left(s, \bar{R}_{m}(s)\right)}\right) \rho\left(s, \bar{R}_{m}(s)\right)\right] d s \mid \\
& +\mid 4 \pi \int_{0}^{t} \bar{R}_{m}(s) \bar{M}_{m}(s)\left[e^{(\bar{\lambda}+\bar{\mu})\left(s, \bar{R}_{m}(s)\right)}\left(\jmath\left(s, \bar{R}_{m}(s)\right)-\overline{\bar{\jmath}}\left(s, \bar{R}_{m}(s)\right)\right)\right. \\
& \left.-\dot{\bar{R}}_{m}(s) e^{2 \bar{\lambda}\left(s, \bar{R}_{m}(s)\right)}\left(\rho\left(s, \bar{R}_{m}(s)\right)-\overline{\bar{\rho}}\left(s, \bar{R}_{m}(s)\right)\right)\right] d s \mid \\
& +\mid 4 \pi \int_{0}^{t} \bar{R}_{m}(s) \bar{M}_{m}(s)\left[e^{(\bar{\lambda}+\bar{\mu})\left(s, \bar{R}_{m}(s)\right)}\left(\overline{\bar{\jmath}}\left(s, \bar{R}_{m}(s)\right)-\bar{\jmath}\left(s, \bar{R}_{m}(s)\right)\right)\right. \\
& \left.-\dot{\bar{R}}_{m}(s) e^{2 \bar{\lambda}\left(s, \bar{R}_{m}(s)\right)}\left(\overline{\bar{\rho}}\left(s, \bar{R}_{m}(s)\right)-\bar{\rho}\left(s, \bar{R}_{m}(s)\right)\right)\right] d s \mid
\end{aligned}
$$

The troublesome term is the last one containing the differences $\overline{\bar{\jmath}}-\bar{\jmath}$ and $\overline{\bar{\rho}}-\bar{\rho}$. If we denote it by $F_{4}$, then

$$
\begin{gathered}
\left|F_{1}\right| \leq C \varepsilon^{3} \int_{0}^{t}\left[\|m(s)-\bar{m}(s)\|_{\infty}+\|\mu(s)-\bar{\mu}(s)\|_{\infty}+\|\lambda(s)-\bar{\lambda}(s)\|_{\infty}\right. \\
\left.+\|\rho(s)-\overline{\bar{\rho}}(s)\|_{\infty}+\|\jmath(s)-\overline{\bar{\jmath}}(s)\|_{\infty}\right] d s+\left|F_{4}\right| .
\end{gathered}
$$

To continue we introduce some abbreviations:

$$
h_{m}(s):=\frac{e^{2 \bar{\lambda}\left(s, \bar{R}_{m}(s)\right)}}{\bar{R}_{m}(s)} \bar{M}_{m}(s), g_{n}(s):=e^{(\mu-\lambda)\left(s, R_{n}(s)\right)}, \bar{g}_{n}(s):=e^{(\bar{\mu}-\bar{\lambda})\left(s, R_{n}(s)\right)}
$$

The definitions of $g_{\bar{n}}$ and $\bar{g}_{\bar{n}}$ are analogous to those of $g_{n}$ and $\bar{g}_{n}$ with $\bar{R}_{n}$ instead of $R_{n}$, and in particular

$$
\frac{W_{n}(s)}{E_{n}(s)} g_{n}(s)=\dot{R}_{n}(s), \frac{\bar{W}_{n}(s)}{\bar{E}_{n}(s)} \bar{g}_{\bar{n}}(s)=\dot{\bar{R}}_{n}(s) .
$$


Moreover, $H_{m, n}^{\delta}(s):=\chi_{\delta}\left(R_{m}(s)-R_{n}(s)\right)$. The definitions of $H_{\bar{m}, n}, H_{\bar{m}}, \bar{n}$, and $H_{m, \bar{n}}^{\delta}$ should be obvious. Inserting these abbreviations and the definition of the source terms into $F_{4}$ yields

$$
\begin{aligned}
& \left|F_{4}\right|=\mid \frac{1}{\delta} \int_{0}^{t} \sum_{n=1}^{N} h_{m}(s)\left[\bar{g}_{\bar{m}}(s)\left(W_{n} M_{n} H \frac{\delta}{m, n}-\bar{W}_{n} \bar{M}_{n} H \frac{\delta}{m}, \bar{n}\right)(s)\right. \\
& \left.-\dot{\bar{R}}_{m}(s)\left(E_{n} M_{n} H_{\bar{m}, n}^{\delta}-\bar{E}_{n} \bar{M}_{n} H_{\bar{m}, \bar{n}}^{\delta}\right)(s)\right] d s \mid \\
& \leq\left|\frac{1}{\delta} \int_{0}^{t} \sum_{n=1}^{N} h_{m}(s) \bar{W}_{n}(s) \bar{M}_{n}(s) H_{\bar{m}, n}^{\delta}(s)\left(\bar{g}_{\bar{n}}-\bar{g}_{n}\right)(s) d s\right| \\
& +\left|\frac{1}{\delta} \int_{0}^{t} \sum_{n=1}^{N} h_{m}(s) \bar{g}_{\bar{m}}(s) H_{\bar{m}, n}^{\delta}(s)\left(W_{n} M_{n}-\bar{W}_{n} \bar{M}_{n}\right)(s) d s\right| \\
& +\left|\frac{1}{\delta} \int_{0}^{t} \sum_{n=1}^{N} h_{m}(s) \bar{M}_{n}(s) H_{\bar{m}, n}^{\delta}(s)\left(\bar{g}_{n} \bar{W}_{n}-g_{n} W_{n} \frac{\bar{E}_{n}}{E_{n}}\right)(s) d s\right| \\
& +\left|\frac{1}{\delta} \int_{0}^{t} \sum_{n=1}^{N} h_{m}(s) \dot{\bar{R}}_{m}(s) H_{\bar{m}, n}^{\delta}(s)\left(\bar{E}_{n} \bar{M}_{n}-E_{n} M_{n}\right)(s) d s\right| \\
& +\left|\frac{1}{\delta} \int_{0}^{t} \sum_{n=1}^{N} h_{m}(s) \bar{W}_{n}(s) \bar{M}_{n}(s)\left(\bar{g}_{\bar{m}}-\bar{g}_{\bar{n}}\right)(s)\left(H \frac{\delta}{\bar{m}, n}-H \frac{\delta}{m}, \bar{n}\right)(s) d s\right| \\
& +\mid \frac{1}{\delta} \int_{0}^{t} \sum_{n=1}^{N} h_{m}(s) \bar{E}_{n}(s) \bar{M}_{n}(s) \\
& \left(H \frac{\delta}{m, n}(s)\left(\dot{R}_{n}-\dot{\bar{R}}_{m}\right)(s)-H \frac{\delta}{m, n}(s)\left(\dot{\bar{R}}_{n}-\dot{\bar{R}}_{m}\right)(s)\right) d s \mid \\
& =: F_{5}+F_{6}+F_{7}+F_{8}+F_{9}+F_{10} .
\end{aligned}
$$

Let $I_{1}(s):=\left\{n \in \mathcal{N}|| R_{n}(s)-\bar{R}_{m}(s) \mid \leq \delta\right\}, I_{2}(s):=\left\{n \in \mathcal{N}|| \bar{R}_{n}(s)-\bar{R}_{m}(s) \mid \leq \delta\right\}$. Then by Lemma 3.6, $\left|I_{1}(s)\right| \leq C \varepsilon^{-3} \delta$ and $\sum_{n \in I_{2}(s)} \bar{M}_{n}(s) \leq C \delta$. Hence,

$$
\begin{aligned}
&\left|F_{5}\right| \leq C \frac{\varepsilon^{6}}{\delta} \int_{0}^{t} \sum_{n \in I_{1}(s)}\left|R_{n}(s)-\bar{R}_{n}(s)\right| d s \leq C \varepsilon^{3} \int_{0}^{t}\|R(s)-\bar{R}(s)\| d s \\
&\left|F_{6}\right| \leq C \frac{\varepsilon^{6}}{\delta} \int_{0}^{t} \sum_{n \in I_{1}(s)}\left(\left|W_{n}(s)-\bar{W}_{n}(s)\right|+\varepsilon^{-3}\left|M_{n}(s)-\bar{M}_{n}(s)\right|\right) d s \\
& \leq C \varepsilon^{3} \int_{0}^{t}(\|W(s)-\bar{W}(s)\|+\|M(s)-\bar{M}(s)\|) d s \\
&\left|F_{7}\right| \leq C \frac{\varepsilon^{6}}{\delta} \int_{0}^{t} \sum_{n \in I_{1}(s)}\left(\|\mu(s)-\bar{\mu}(s)\|_{\infty}+\|\lambda(s)-\bar{\lambda}(s)\|_{\infty}\right. \\
&\left.\quad+\left|R_{n}(s)-\bar{R}_{n}(s)\right|+\left|W_{n}(s)-\bar{W}_{n}(s)\right|\right) d s
\end{aligned}
$$




$$
\begin{gathered}
\leq C \varepsilon^{3} \int_{0}^{t}\left(\|\mu(s)-\bar{\mu}(s)\|_{\infty}+\|\lambda(s)-\bar{\lambda}(s)\|_{\infty}\right. \\
+\|R(s)-\bar{R}(s)\|+\|W(s)-\bar{W}(s)\|) d s, \\
\left|F_{8}\right| \leq C \frac{\varepsilon^{6}}{\delta} \int_{0}^{t} \sum_{n \in I_{1}(s)}\left(\left|R_{n}(s)-\bar{R}_{n}(s)\right|+\left|W_{n}(s)-\bar{W}_{n}(s)\right|\right. \\
\left.\quad+\varepsilon^{-3}\left|M_{n}(s)-\bar{M}_{n}(s)\right|\right) d s \\
\leq C \varepsilon^{3} \int_{0}^{t}(\|R(s)-\bar{R}(s)\|+\|W(s)-\bar{W}(s)\|+\|M(s)-\bar{M}(s)\|) d s, \\
\left|F_{9}\right| \leq C \frac{\varepsilon^{3}}{\delta} \int_{0}^{t} \sum_{n \in I_{1}(s) \cup I_{2}(s)} \bar{M}_{n}(s)\left|\bar{R}_{m}(s)-\bar{R}_{n}(s)\right| \\
\leq C \frac{\varepsilon^{3}}{\delta} \int_{0}^{t} \sum_{n \in I_{1}(s) \cup I_{2}(s)} \overline{M i n}_{n}(s)(\delta+\|R(s)-\bar{R}(s)\|) \\
\leq C \frac{\varepsilon^{3}}{\delta} \int_{0}^{t} \sum_{n} \min \{\|R(s)-\bar{R}(s)\| / \delta, 1\} d s \\
\leq C \varepsilon^{3} \int_{0}^{t}\|R(s)-\bar{R}(s)\| \sum_{n \in I_{1}(s) \cup I_{2}(s)} \bar{M}_{n}(s) d s
\end{gathered}
$$

To deal with $F_{10}$ let $g_{n, m}(s):=h_{m}(s) \bar{E}_{n}(s) \bar{M}_{n}(s)$. By Lemma 3.5, $\left|\dot{\bar{M}}_{n}\right| \leq C \varepsilon^{3}$, hence for all $n, m \in \mathcal{N}$,

$$
\left\|g_{n, m}\right\|_{\infty} \leq C \varepsilon^{6}, \quad\left\|\dot{g}_{n, m}\right\|_{\infty} \leq C \varepsilon^{6} .
$$

Thus Lemma 3.12 yields

$$
\begin{aligned}
\left|F_{10}\right| & \leq C \varepsilon^{-3} \max _{k, l \in \mathcal{N}}\left(\left\|g_{k, l}\right\|_{\infty}+\left\|\dot{g}_{k, l}\right\|_{\infty}\right)\|R(t)-\bar{R}(t)\| \\
& \leq C \varepsilon^{3}\|R(t)-\bar{R}(t)\| .
\end{aligned}
$$

Putting all our estimates together and observing Lemma 3.7, we get

$$
\frac{\left|\overline{\bar{M}}_{m}(t)-\bar{M}_{m}(t)\right|}{\varepsilon^{3}} \leq C \int_{0}^{t} \frac{\left|\overline{\bar{M}}_{m}(s)-\bar{M}_{m}(s)\right|}{\varepsilon^{3}} d s+C \int_{0}^{t} e(s) d s+C\|R(t)-\bar{R}(t)\| .
$$

Taking the maximum over $m \in \mathcal{N}$ and using a Gronwall argument completes the proof.

Lemma 3.14 With e(s) defined as in Lemma 3.19,

$$
\|W(t)-\bar{W}(t)\| \leq C \int_{0}^{t} e(s) d s+C\|R(t)-\bar{R}(t)\|, t \in\left[0, T_{\varepsilon, \delta}\right]
$$


Proof. The proof is similar to the one of Lemma 3.13. Let $t \in\left[0, T_{\varepsilon, \delta}\right]$ and $m \in \mathcal{N}$. From the differential equations for $W_{m}$ and $W_{m}$,

$$
\begin{aligned}
W_{m}(t)-\bar{W}_{m}(t)=\int_{0}^{t} & \frac{e^{(\mu-\lambda)\left(s, R_{m}(s)\right)} L_{m}}{R_{m}^{3}(s) E_{m}(s)}-\dot{\lambda}\left(s, R_{m}(s)\right) W_{m}(s) \\
& -e^{(\mu-\lambda)\left(s, R_{m}(s)\right)} \mu^{\prime}\left(s, R_{m}(s)\right) E_{m}(s)-\frac{e^{(\bar{\mu}-\bar{\lambda})\left(s, \bar{R}_{m}(s)\right)} L_{m}}{\bar{R}_{m}^{3}(s) \bar{E}_{m}(s)} \\
+ & \left.\bar{\lambda}\left(s, \bar{R}_{m}(s)\right) \bar{W}_{m}(s)+e^{(\bar{\mu}-\bar{\lambda})\left(s, \bar{R}_{m}(s)\right)} \bar{\mu}^{\prime}\left(s, \bar{R}_{m}(s)\right) \bar{E}_{m}(s)\right] d s .
\end{aligned}
$$

Inserting the equations for $\dot{\lambda}, \mu^{\prime}, \overline{\dot{\lambda}}$ and $\bar{\mu}^{\prime}$ yields

$$
\begin{aligned}
& W_{m}(t)-\bar{W}_{m}(t)= \\
& \int_{0}^{t}\left[\frac{e^{(\mu-\lambda)\left(s, R_{m}(s)\right)} L_{m}}{R_{m}^{3}(s) E_{m}(s)}-\frac{e^{(\bar{\mu}-\bar{\lambda})\left(s, \bar{R}_{m}(s)\right)} L_{m}}{\bar{R}_{m}^{3}(s) \bar{E}_{m}(s)}\right] d s \\
& +\int_{0}^{t}\left[e^{(\bar{\mu}+\bar{\lambda})\left(s, \bar{R}_{m}(s)\right)} \bar{E}_{m}(s) \frac{\bar{m}\left(s, \bar{R}_{m}(s)\right)}{\bar{R}_{m}^{2}(s)}-e^{(\mu+\lambda)\left(s, R_{m}(s)\right)} E_{m}(s) \frac{m\left(s, R_{m}(s)\right)}{R_{m}^{2}(s)}\right] d s \\
& +\int_{0}^{t}\left[4 \pi R_{m}(s) e^{(\mu+\lambda)\left(s, R_{m}(s)\right)}\left(W_{m}(s) \jmath\left(s, R_{m}(s)\right)-E_{m}(s) p\left(s, R_{m}(s)\right)\right)\right. \\
& \left.\quad-4 \pi \bar{R}_{m}(s) e^{(\bar{\mu}+\bar{\lambda})\left(s, \bar{R}_{m}(s)\right)}\left(\bar{W}_{m}(s) \bar{\jmath}\left(s, \bar{R}_{m}(s)\right)-\bar{E}_{m}(s) \bar{p}\left(s, \bar{R}_{m}(s)\right)\right)\right] d s \\
& =: F_{1}+F_{2}+F_{3} ;
\end{aligned}
$$

we have changed the order of terms to group the un-integrated source terms together. Using the same calculations as in the lemmas above we find

$$
\begin{aligned}
& \left|F_{1}\right| \leq C \int_{0}^{t}\left(\|\mu(s)-\bar{\mu}(s)\|_{\infty}+\|\lambda(s)-\bar{\lambda}(s)\|_{\infty}\right. \\
& +\|R(s)-\bar{R}(s)\|+\|W(s)-\bar{W}(s)\|) d s, \\
& \left|F_{2}\right| \leq C \int_{0}^{t}\left(\|\mu(s)-\bar{\mu}(s)\|_{\infty}+\|\lambda(s)-\bar{\lambda}(s)\|_{\infty}+\|m(s)-\bar{m}(s)\|_{\infty}\right. \\
& +\|R(s)-\bar{R}(s)\|+\|W(s)-\bar{W}(s)\|) d s, \\
& \left|F_{3}\right| \leq 4 \pi \mid \int_{0}^{t}\left[R_{m}(s) e^{(\mu+\lambda)\left(s, R_{m}(s)\right)}\left(W_{m}(s) \jmath\left(s, R_{m}(s)\right)-E_{m}(s) p\left(s, R_{m}(s)\right)\right)\right. \\
& \left.-\bar{R}_{m}(s) e^{(\bar{\mu}+\bar{\lambda})\left(s, \bar{R}_{m}(s)\right)}\left(\bar{W}_{m}(s) \jmath\left(s, \bar{R}_{m}(s)\right)-\bar{E}_{m}(s) p\left(s, \bar{R}_{m}(s)\right)\right)\right] d s \mid \\
& +4 \pi \mid \int_{0}^{t} \bar{R}_{m}(s) e^{(\bar{\mu}+\bar{\lambda})\left(s, \bar{R}_{m}(s)\right)}\left[\bar{W}_{m}(s)\left(\jmath\left(s, \bar{R}_{m}(s)\right)-\overline{\bar{\jmath}}\left(s, \bar{R}_{m}(s)\right)\right)\right. \\
& \left.-\bar{E}_{m}(s)\left(p\left(s, \bar{R}_{m}(s)\right)-\overline{\bar{p}}\left(s, \bar{R}_{m}(s)\right)\right)\right] d s \mid
\end{aligned}
$$




$$
\begin{aligned}
& +4 \pi \mid \int_{0}^{t} \bar{R}_{m}(s) e^{(\bar{\mu}+\bar{\lambda})\left(s, \bar{R}_{m}(s)\right)}\left[\bar{W}_{m}(s)\left(\overline{\bar{\jmath}}\left(s, \bar{R}_{m}(s)\right)-\bar{\jmath}\left(s, \bar{R}_{m}(s)\right)\right)\right. \\
& \left.-\bar{E}_{m}(s)\left(\overline{\bar{p}}\left(s, \bar{R}_{m}(s)\right)-\bar{p}\left(s, \bar{R}_{m}(s)\right)\right)\right] d s \mid .
\end{aligned}
$$

Denote the last term - the one with the differences $\overline{\bar{\jmath}}-\bar{\jmath}$ and $\overline{\bar{p}}-\bar{p}$-by $F_{4}$. Then

$$
\begin{aligned}
\left|F_{3}\right| \leq C \int_{0}^{t}\left[\|\mu(s)-\bar{\mu}(s)\|_{\infty}+\|\lambda(s)-\bar{\lambda}(s)\|_{\infty}+\|R(s)-\bar{R}(s)\|\right. \\
\left.\quad+\|W(s)-\bar{W}(s)\|+\|p(s)-\overline{\bar{p}}(s)\|_{\infty}+\|\jmath(s)-\overline{\bar{\jmath}}(s)\|_{\infty}\right] d s+\left|F_{4}\right| .
\end{aligned}
$$

We insert the definitions of the source terms into $F_{4}$, define $k_{m}(s):=$ $e^{2 \bar{\lambda}\left(s, \bar{R}_{m}(s)\right)} \bar{E}_{m}(s) / \bar{R}_{m}(s)$, recall the definitions of $g_{m}, H_{m, n}^{\delta}$ etc. from the proof of Lemma 3.13 and note that $\bar{W}_{m} \bar{g}_{\bar{m}} / \bar{E}_{m}=\dot{\bar{R}}_{m}$. Then

$$
\begin{aligned}
\left|F_{4}\right|=\mid \frac{1}{\delta} \int_{0}^{t} \sum_{n=1}^{N} k_{m}(s) \bar{g}_{\bar{m}}(s)\left[\frac{\bar{W}_{m}(s)}{\bar{E}_{m}(s)}\left(W_{n} M_{n} H_{\bar{m}, n}^{\delta}-\bar{W}_{n} \bar{M}_{n} H_{\bar{m}, \bar{n}}^{\delta}\right)(s)\right. \\
\left.+\left(\frac{\bar{W}_{n}^{2}}{\bar{E}_{n}} \bar{M}_{n} H_{\frac{\delta}{m}, \bar{n}}-\frac{W_{n}^{2}}{E_{n}} M_{n} H_{\bar{m}, n}^{\delta}\right)(s)\right] d s \mid \\
\leq\left|\frac{1}{\delta} \int_{0}^{t} \sum_{n=1}^{N} k_{m}(s) \bar{M}_{n}(s) H_{\bar{m}, n}(s)\left(\frac{\bar{W}_{n} W_{n}}{E_{n}} g_{n}-\frac{\bar{W}_{n} \bar{W}_{n}}{\bar{E}_{n}} \bar{g}_{\bar{n}}\right)(s) d s\right| \\
+\left|\frac{1}{\delta} \int_{0}^{t} \sum_{n=1}^{N} k_{m}(s) \frac{\bar{W}_{m}(s)}{\bar{E}_{m}(s)} \bar{g}_{\bar{m}}(s) H_{\bar{m}, n}(s)\left(W_{n} M_{n}-\bar{W}_{n} \bar{M}_{n}\right)(s) d s\right| \\
+\left|\frac{1}{\delta} \int_{0}^{t} \sum_{n=1}^{N} k_{m}(s) \bar{g}_{\bar{m}}(s) H_{\bar{m}, n}(s)\left(\frac{\bar{W}_{n}^{2}}{\bar{E}_{n}} \bar{M}_{n}-\frac{W_{n}^{2}}{E_{n}} M_{n}\right)(s) d s\right| \\
+\left|\frac{1}{\delta} \int_{0}^{t} \sum_{n=1}^{N} k_{m}(s) \frac{\bar{W}_{n}^{2}(s)}{\bar{E}_{n}(s)} \bar{M}_{n}(s)\left(\bar{g}_{\bar{m}}-\bar{g}_{\bar{n}}\right)(s)\left(H_{\bar{m}, \bar{n}}-H_{\bar{m}, n}\right)(s) d s\right| \\
+\mid \frac{1}{\delta} \int_{0}^{t} \sum_{n=1}^{N} k_{m}(s) \bar{W}_{n}(s) \bar{M}_{n}(s) \\
=: F_{5}+F_{6}+F_{7}+F_{8}+F_{9} .
\end{aligned}
$$

Let $I_{1}(s):=\left\{n \in \mathcal{N}|| R_{n}(s)-\bar{R}_{m}(s) \mid \leq \delta\right\}, I_{2}(s):=\left\{n \in \mathcal{N}|| \bar{R}_{n}(s)-\bar{R}_{m}(s) \mid \leq \delta\right\}$. Then by Lemma 3.6, $\left|I_{1}(s)\right| \leq C \varepsilon^{-3} \delta$ and $\sum_{n \in I_{2}(s)} \bar{M}_{n}(s) \leq C \delta$. Hence,

$$
\left|F_{5}\right| \leq C \int_{0}^{t}\left(\|\mu(s)-\bar{\mu}(s)\|_{\infty}+\|\lambda(s)-\bar{\lambda}(s)\|_{\infty}\right.
$$




$$
\begin{aligned}
&+\|R(s)-\bar{R}(s)\|+\|W(s)-\bar{W}(s)\|) d s, \\
&\left|F_{6}\right| \leq C \frac{\varepsilon^{3}}{\delta} \int_{0}^{t} \sum_{n \in I_{1}(s)}\left(\left|W_{n}(s)-\bar{W}_{n}(s)\right|+\varepsilon^{-3}\left|M_{n}(s)-\bar{M}_{n}(s)\right|\right) d s \\
& \leq C \int_{0}^{t}(\|W(s)-\bar{W}(s)\|+\|M(s)-\bar{M}(s)\|) d s, \\
&\left|F_{7}\right| \leq C \frac{\varepsilon^{3}}{\delta} \int_{0}^{t} \sum_{n \in I_{1}(s)}\left(\left|R_{n}-\bar{R}_{n}\right|+\left|W_{n}-\bar{W}_{n}\right|+\varepsilon^{-3}\left|M_{n}-\bar{M}_{n}\right|\right)(s) d s \\
& \leq C \int_{0}^{t}(\|R(s)-\bar{R}(s)\|+\|W(s)-\bar{W}(s)\|+\|M(s)-\bar{M}(s)\|) d s, \\
&\left|F_{8}\right| \leq \frac{C}{\delta} \int_{0}^{t} \sum_{n \in I_{1}(s) \cup I_{2}(s)} \bar{M}_{n}(s)\left(\left|\bar{R}_{m}-\bar{R}_{n}\right| \min \left\{\left|R_{n}-\bar{R}_{n}\right| / \delta, 1\right\}\right)(s) d s \\
& \leq \frac{C}{\delta} \int_{0}^{t} \sum_{n \in I_{1}(s) \cup I_{2}(s)} \bar{M}_{n}(s)((\delta+\|R-\bar{R}\|) \min \{\|R-\bar{R}\| / \delta, 1\})(s) d s \\
& \leq C \int_{0}^{t}\|R(s)-\bar{R}(s)\| d s .
\end{aligned}
$$

Finally, let $g_{n, m}(s):=k_{m}(s) \bar{W}_{n}(s) \bar{M}_{n}(s)$. By Lemma $3.5,\left|\dot{\bar{M}}_{n}(s)\right| \leq C \varepsilon^{3}$, hence

$$
\left\|g_{n, m}\right\|_{\infty} \leq C \varepsilon^{3},\left\|\dot{g}_{n, m}\right\|_{\infty} \leq C \varepsilon^{3}, n, m \in \mathcal{N} .
$$

Thus Lemma 3.12 yields

$$
\left|F_{9}\right| \leq C \varepsilon^{-3} \max _{k, l \in \mathcal{N}}\left(\left\|g_{k, l}\right\|_{\infty}+\left\|\dot{g}_{k, l}\right\|_{\infty}\right)\|R(t)-\bar{R}(t)\| \leq C\|R(t)-\bar{R}(t)\| .
$$

Collecting all these estimates and recalling Lemma 3.7 completes the proof.

We are finally ready to prove our main results:

Proof of Theorem 2.2 and Theorem 3.2 .

By Lemma 3.13,

$$
\begin{aligned}
\|\bar{M}(t)-\overline{\bar{M}}(t)\| \leq & C(\|R(t)-\bar{R}(t)\|+\Delta) \\
& +C \int_{0}^{t}(\|W(s)-\bar{W}(s)\|+\|M(s)-\bar{M}(s)\|) d s .
\end{aligned}
$$

where

$$
\Delta:=\sup _{t \in\left[0, T_{\varepsilon, \delta}\right]}\left(\|\rho(t)-\overline{\bar{\rho}}(t)\|_{\infty}+\|p(t)-\overline{\bar{p}}(t)\|_{\infty}+\left\|_{\jmath}(t)-\overline{\bar{\jmath}}(t)\right\|_{\infty}\right) .
$$

Combining this with Lemma 3.10 yields

$$
\begin{aligned}
\|M(t)-\bar{M}(t)\| \leq & C(\|R(t)-\bar{R}(t)\|+\Delta+\varepsilon) \\
& +C \int_{0}^{t}(\|W(s)-\bar{W}(s)\|+\|M(s)-\bar{M}(s)\|) d s .
\end{aligned}
$$


Next, by Lemma 3.14,

$$
\begin{aligned}
\|W(t)-\bar{W}(t)\| \leq & C(\|R(t)-\bar{R}(t)\|+\Delta) \\
& +\int_{0}^{t}(\|W(s)-\bar{W}(s)\|+\|M(s)-\bar{M}(s)\|) d s .
\end{aligned}
$$

Adding the last two estimates and applying a Gronwall argument implies

$$
\|W(t)-\bar{W}(t)\|+\|M(t)-\bar{M}(t)\| \leq C(\|R(t)-\bar{R}(t)\|+\Delta+\varepsilon) .
$$

Using Lemma 3.11 and Lemma 3.7 we find

$$
\begin{aligned}
\left|R_{n}(t)-\bar{R}_{n}(t)\right| \leq & C \int_{0}^{t}\left(\left|R_{n}(s)-\bar{R}_{n}(s)\right|+\left|W_{n}(s)-\bar{W}_{n}(s)\right|\right. \\
\leq & \left.C \Delta \quad+\|\mu(s)-\bar{\mu}(s)\|_{\infty}+\|\lambda(s)-\bar{\lambda}(s)\|_{\infty}\right) d s \\
& +C \int_{0}^{t}(\|R(s)-\bar{R}(s)\|+\|W(s)-\bar{W}(s)\|+\|M(s)-\bar{M}(s)\|) d s
\end{aligned}
$$

for all $t \in\left[0, T_{\varepsilon, \delta}\right]$ and $n \in \mathcal{N}$. Taking the maximum over $n \in \mathcal{N}$ and inserting (3.5) yields

$$
\|R(t)-\bar{R}(t)\| \leq C\left(\Delta+\varepsilon+\int_{0}^{t}\|R(s)-\bar{R}(s)\| d s\right)
$$

which via Gronwall implies that

$$
\|R(t)-\bar{R}(t)\| \leq C(\Delta+\varepsilon) .
$$

Now we apply Lemma 3.8:

$$
\Delta \leq C\left\{\begin{array}{cc}
\delta+\varepsilon / \delta & \text { in case of Theorem 3.2, } \\
\delta^{2}+\varepsilon / \delta & \text { in case of Theorem 2.2 }
\end{array}\right.
$$

Inserting this into (3.6) proves the corresponding error estimates on $\| R(t)-$ $\bar{R}(t) \|$ in the two theorems. The error estimates for $\|W(t)-\bar{W}(t)\|$ and $\|M(t)-\bar{M}(t)\|$ follow from (3.5), and those for $\|\mu(t)-\bar{\mu}(t)\|_{\infty},\|\lambda(t)-\bar{\lambda}(t)\|_{\infty}$, and $\|m(t)-\bar{m}(t)\|_{\infty}$ follow from Lemma 3.7. Under the assumption of Theorem 2.2 the estimates for $\|\rho(t)-\bar{\rho}(t)\|_{\infty},\|p(t)-\bar{p}(t)\|_{\infty}$ and $\|\jmath(t)-\bar{\jmath}(t)\|_{\infty}$ are valid due to Lemma 3.8 and Lemma 3.9 .

It remains to establish the assertions on the length $T_{\varepsilon, \delta}$ of our approximation interval. Let $C$ denote a constant for which the error estimates in Theorem 2.2 or Theorem 3.2 hold; note that $C$ is independent of $\delta$ and $\varepsilon$. For Theorem 3.2, let $\delta$ be so small that

$$
\delta+\frac{\varepsilon}{\delta} \leq \frac{1}{32 D^{2} C} .
$$


Then

$$
\begin{aligned}
\bar{R}_{n}(t) & \leq R_{n}(t)+\|R(t)-\bar{R}(t)\| \leq D+C\left(\delta+\frac{\varepsilon}{\delta}\right) \leq \frac{3}{2} D, \\
\frac{1}{\bar{R}_{n}(t)} & =\frac{1}{R_{n}(t)}+\frac{R_{n}(t)-\bar{R}_{n}(t)}{R_{n}(t) \bar{R}_{n}(t)} \leq D+2 D^{2} C\left(\delta+\frac{\varepsilon}{\delta}\right) \leq \frac{3}{2} D, \\
\left|\bar{W}_{n}(t)\right| & \leq\left|W_{n}(t)\right|+\|W(t)-\bar{W}(t)\| \leq D+C\left(\delta+\frac{\varepsilon}{\delta}\right) \leq \frac{3}{2} D, \\
\left|\bar{M}_{n}(t)\right| & \leq\left|M_{n}(t)\right|+\varepsilon^{3}\|M(t)-\bar{M}(t)\| \leq \varepsilon^{3}\left(D+C\left(\delta+\frac{\varepsilon}{\delta}\right)\right) \leq \varepsilon^{3} \frac{3}{2} D, \\
e^{2 \bar{\lambda}(t, r)} & =e^{2 \lambda(t, r)}+\frac{2}{r}(\bar{m}-m)(t, r) e^{2(\lambda+\bar{\lambda})(t, r)} \leq D+16 D^{3} C\left(\delta+\frac{\varepsilon}{\delta}\right) \leq \frac{3}{2} D
\end{aligned}
$$

for all $t \in\left[0, T_{\varepsilon, \delta}\right]$ and $r>0$ since $\bar{m}(t, r)=m(t, r)=0$ for $r<1 /(4 D)$. Thus $T_{\varepsilon, \delta}=$ $T$ since otherwise the approximation interval could be extended beyond $T_{\varepsilon, \delta}$.

For Theorem 2.2, let $\delta$ be so small that

$$
\delta+\frac{\varepsilon}{\delta} \leq \frac{1}{32 D^{2} C} \wedge \delta+\frac{\varepsilon}{\delta^{2}} \leq \frac{D}{2 C} .
$$

Then the above inequalities hold for all $t \in\left[0, T_{\varepsilon, \delta}\right]$, and in addition

$$
\|\bar{\rho}(t)\|_{\infty} \leq\|\rho(t)\|_{\infty}+\|\bar{\rho}(t)-\rho(t)\|_{\infty} \leq D+C\left(\delta+\frac{\varepsilon}{\delta^{2}}\right) \leq \frac{3}{2} D
$$

With the same argument as above, $T_{\varepsilon, \delta}=T$. This completes the proof of the main results.

\section{The fully discretized approximation}

In setting up a discretized version of a system like the Vlasov-Einstein system discretizing the phase space is the major step. For example, in several of the papers on numerical schemes for the Vlasov-Poisson or Vlasov-Maxwell systems mentioned in the introduction only this step is analyzed. However, due to the presence of the un-integrated source terms in the characteristic system, we can not discretize our system in time as easily. We have to modify the evolution equations slightly in order to get a convergence result, the idea being to discretize after integrating by parts in Lemma 3.12. Recall that the latter maneuver was essential for the convergence proof of the semi-discretized approximation, and we have to set up things in such a way that there is a substitute for this in the fully discretized case.

Throughout this section the assumptions of Theorem 2.2 are to be satisfied. Moreover, we assume $\varepsilon \leq \delta^{2}$ and $\delta \leq 1 /(8 D)$. Let $0<\tau \ll 1$ be a small parameter, the time step. Before we discretize the evolution equations for $\bar{R}_{n}(t), \bar{W}_{n}(t), \bar{M}_{n}(t)$ we regroup the terms in these equations as follows; only the equations for $\bar{W}_{n}(t)$ and $\bar{M}_{n}(t)$ need to be considered, the discretization of 
the equation for $\bar{R}_{n}(t)$ being trivial:

$$
\begin{aligned}
\dot{\bar{W}}_{n}(t)= & e^{(\bar{\mu}-\bar{\lambda})\left(t, \bar{R}_{n}(t)\right)} \frac{L_{n}}{\bar{R}_{n}^{3}(t) \bar{E}_{n}(t)}-e^{(\bar{\mu}+\bar{\lambda})\left(t, \bar{R}_{n}(t)\right)} \frac{\bar{m}\left(t, \bar{R}_{n}(t)\right)}{\bar{R}_{n}^{2}(t)} \bar{E}_{n}(t) \\
+ & \frac{e^{2 \bar{\lambda}\left(t, \bar{R}_{n}(t)\right)}}{\bar{R}_{n}(t)} 4 \pi \bar{R}_{n}^{2}(t) e^{(\bar{\mu}-\bar{\lambda})\left(t, \bar{R}_{n}(t)\right)}\left(\bar{W}_{n}(t) \bar{\jmath}\left(t, \bar{R}_{n}(t)\right)-\bar{E}_{n}(t) \bar{p}\left(t, \bar{R}_{n}(t)\right)\right) \\
= & e^{(\bar{\mu}-\bar{\lambda})\left(t, \bar{R}_{n}(t)\right)} \frac{L_{n}}{\bar{R}_{n}^{3}(t) \bar{E}_{n}(t)}-e^{(\bar{\mu}+\bar{\lambda})\left(t, \bar{R}_{n}(t)\right)} \frac{\bar{m}\left(t, \bar{R}_{n}(t)\right)}{\bar{R}_{n}^{2}(t)} \bar{E}_{n}(t) \\
& +\frac{e^{2 \bar{\lambda}\left(t, \bar{R}_{n}(t)\right)}}{\bar{R}_{n}(t)} \frac{1}{\delta} \sum_{m=1}^{N} \bar{M}_{m}(t) \bar{W}_{m}(t) \bar{E}_{n}(t) \frac{d}{d t} \chi\left(\bar{R}_{n}(t)-\bar{R}_{m}(t)\right) \\
& +\frac{e^{2 \bar{\lambda}\left(t, \bar{R}_{n}(t)\right)}}{\bar{R}_{n}(t)} \frac{1}{\delta} \sum_{m=1}^{N} \bar{M}_{m}(t) \bar{W}_{m}^{2}(t) \frac{\bar{E}_{n}(t)}{\bar{E}_{m}(t)} \chi \delta\left(\bar{R}_{n}(t)-\bar{R}_{m}(t)\right) \\
= & : \overline{F W}_{n}^{(}(t)+\overline{F W}_{n}^{2}(t)+\overline{F W}_{n}^{3}(t) ;
\end{aligned}
$$

note that

$$
\begin{aligned}
\frac{d}{d t} \chi\left(\bar{R}_{n}(t)-\bar{R}_{m}(t)\right)= & \frac{1}{\delta} \chi_{\delta}\left(\bar{R}_{n}(t)-\bar{R}_{m}(t)\right) \\
& \left(e^{(\bar{\mu}-\bar{\lambda})\left(t, \bar{R}_{n}(t)\right)} \frac{\bar{W}_{n}(t)}{\bar{E}_{n}(t)}-e^{(\bar{\mu}-\bar{\lambda})\left(t, \bar{R}_{m}(t)\right)} \frac{\bar{W}_{m}(t)}{\bar{E}_{m}(t)}\right) .
\end{aligned}
$$

Similarly,

$$
\begin{aligned}
\dot{\bar{M}}_{n}(t)= & \bar{M}_{n}(t)\left[e^{(\bar{\mu}+\bar{\lambda})\left(t, \bar{R}_{n}(t)\right)} \frac{\bar{m}\left(t, \bar{R}_{n}(t)\right)}{\bar{R}_{n}^{2}(t)} \frac{\bar{W}_{n}(t)}{\bar{E}_{n}(t)}\right. \\
& \left.+\frac{e^{2 \lambda\left(t, \bar{R}_{n}(t)\right)}}{\bar{R}_{n}(t)} 4 \pi \bar{R}_{n}^{2}(t) e^{(\bar{\mu}-\bar{\lambda})\left(t, \bar{R}_{n}(t)\right)}\left(\bar{\jmath}\left(t, \bar{R}_{n}(t)\right)-\frac{\bar{W}_{n}(t)}{\bar{E}_{n}(t)} \bar{\rho}\left(t, \bar{R}_{n}(t)\right)\right)\right] \\
= & \bar{M}_{n}(t)\left[e^{(\bar{\mu}+\bar{\lambda})\left(t, \bar{R}_{n}(t)\right)} \frac{\bar{m}\left(t, \bar{R}_{n}(t)\right)}{\bar{R}_{n}^{2}(t)} \frac{\bar{W}_{n}(t)}{\bar{E}_{n}(t)}\right. \\
& -\frac{e^{2 \bar{\lambda}\left(t, \bar{R}_{n}(t)\right)}}{\bar{R}_{n}(t)} \frac{1}{\delta} \sum_{m=1}^{N} \bar{M}_{m}(t) \bar{E}_{m}(t) \frac{d}{d t} \chi\left(\bar{R}_{n}(t)-\bar{R}_{m}(t)\right) \\
& +\frac{e^{2 \bar{\lambda}\left(t, \bar{R}_{n}(t)\right)}}{\bar{R}_{n}(t)} \frac{1}{\delta} \sum_{m=1}^{N} \bar{M}_{m}(t) \bar{W}_{m}(t) \chi_{\delta}\left(\bar{R}_{n}(t)-\bar{R}_{m}(t)\right) \\
=: & \bar{M}_{n}(t)\left[\overline{F M}_{n}^{1}(t)+\overline{F M}_{n}^{2}(t)+\overline{F M}_{n}^{3}(t)\right] .
\end{aligned}
$$


The terms $\overline{F W}_{n}^{1}(t), \overline{F W}_{n}^{2}(t), \overline{F W}_{n}^{3}(t)$ and $\overline{F M}_{n}^{1}(t), \overline{F M}_{n}^{2}(t), \overline{F M}_{n}^{3}(t)$ have natural discretizations given below; note that $\frac{d}{d t} \chi\left(\bar{R}_{n}(t)-\bar{R}_{m}(t)\right)$ is much better to discretize and analyze than the term $\frac{1}{\delta}\left(\dot{\bar{R}}_{n}-\dot{\bar{R}}_{m}\right) \chi_{\delta}\left(\bar{R}_{n}(t)-\bar{R}_{m}(t)\right)$. Discretizing the equations above leads to the following Euler-like scheme:

$$
\begin{aligned}
& \bar{R}_{n, i+1}:=\bar{R}_{n, i}+\tau e^{\left(\bar{\mu}_{i}-\bar{\lambda}_{i}\right)\left(\bar{R}_{n, i}\right)} \frac{\bar{W}_{n, i}}{\bar{E}_{n, i}}, \\
& \bar{W}_{n, i+1}:=\bar{W}_{n, i}+\tau\left(\overline{F W}_{n, i}^{1}+\frac{e^{2 \bar{\lambda}_{i}\left(\bar{R}_{n, i}\right)}}{\bar{R}_{n, i}} \overline{F W}_{n, i}^{2}+\overline{F W}_{n, i}^{3}\right), \\
& \bar{M}_{n, i+1}:=\bar{M}_{n, i}+\tau \bar{M}_{n, i}\left(\overline{F M}_{n, i}^{1}+\frac{e^{2 \bar{\lambda}_{i}\left(\bar{R}_{n, i}\right)}}{\bar{R}_{n, i}} \overline{F M}_{n, i}^{2}+\overline{F M}_{n, i}^{3}\right) \text {, } \\
& \overline{F W}_{n, i}^{1}:=e^{\left(\bar{\mu}_{i}-\bar{\lambda}_{i}\right)\left(\bar{R}_{n, i}\right)} \frac{L_{n}}{\bar{R}_{n, i}^{3} \bar{E}_{n, i}}-e^{\left(\bar{\mu}_{i}+\bar{\lambda}_{i}\right)\left(\bar{R}_{n, i}\right)} \frac{\bar{m}_{i}\left(\bar{R}_{n, i}\right)}{\bar{R}_{n, i}^{2}} \bar{E}_{n, i}, \\
& \overline{F W}_{n, i}^{2}:=\frac{e^{2 \bar{\lambda}_{i}\left(\bar{R}_{n, i}\right)}}{\bar{R}_{n, i}} \sum_{m=1}^{N} \bar{M}_{m, i} \bar{W}_{m, i} \bar{E}_{n, i} \frac{\chi\left(\bar{R}_{n, i+1}-\bar{R}_{m, i+1}\right)-\chi\left(\bar{R}_{n, i}-\bar{R}_{m, i}\right)}{\tau}, \\
& \overline{F W}_{n, i}^{3}:=\frac{e^{2 \bar{\lambda}_{i}\left(\bar{R}_{n, i}\right)}}{\bar{R}_{n, i}} \frac{1}{\delta} \sum_{m=1}^{N} \bar{M}_{m, i} \bar{W}_{m, i}^{2} \frac{\bar{E}_{n, i}}{\bar{E}_{m, i}} \chi_{\delta}\left(\bar{R}_{n, i}-\bar{R}_{m, i}\right) \\
& \left(e^{\left(\bar{\mu}_{i}-\bar{\lambda}_{i}\right)\left(\bar{R}_{m, i}\right)}-e^{\left(\bar{\mu}_{i}-\bar{\lambda}_{i}\right)\left(\bar{R}_{n, i}\right)}\right), \\
& \overline{F M}_{n, i}^{1}:=e^{\left(\bar{\mu}_{i}+\bar{\lambda}_{i}\right)\left(\bar{R}_{n, i}\right)} \frac{\bar{m}_{i}\left(\bar{R}_{n, i}\right)}{\bar{R}_{n, i}^{2}} \frac{\bar{W}_{n, i}}{\bar{E}_{n, i}} \\
& \overline{F M}_{n, i}^{2}:=\frac{e^{2 \bar{\lambda}_{i}\left(\bar{R}_{n, i}\right)}}{\bar{R}_{n, i}} \sum_{m=1}^{N} \bar{M}_{m, i} \bar{E}_{m, i} \frac{\chi\left(\bar{R}_{n, i+1}-\bar{R}_{m, i+1}\right)-\chi\left(\bar{R}_{n, i}-\bar{R}_{m, i}\right)}{\tau}, \\
& \overline{F M}_{n, i}^{3}:=\frac{e^{2 \bar{\lambda}_{i}\left(\bar{R}_{n, i}\right)}}{\bar{R}_{n, i}} \frac{1}{\delta} \sum_{m=1}^{N} \bar{M}_{m, i} \bar{W}_{m, i} \chi_{\delta}\left(\bar{R}_{n, i}-\bar{R}_{m, i}\right) \\
& \left(e^{\left(\bar{\mu}_{i}-\bar{\lambda}_{i}\right)\left(\bar{R}_{n, i}\right)}-e^{\left(\bar{\mu}_{i}-\bar{\lambda}_{i}\right)\left(\bar{R}_{m, i}\right)}\right),
\end{aligned}
$$

with initial data

$$
\left(\bar{R}_{n, 0}, \bar{W}_{n, 0}, \bar{M}_{n, 0}\right):=\left(\bar{R}_{n}(0), \bar{W}_{n}(0), \bar{M}_{n}(0)\right), n \in \mathcal{N} .
$$

We have abbreviated

$$
\bar{E}_{n, i}:=\sqrt{1+\bar{W}_{n, i}^{2}+L_{n} / \bar{R}_{n, i}^{2}} ;
$$

note that $\bar{R}_{n, i+1}$ can be computed without knowing the terms $\overline{F W}_{n, i}^{1}$ and $\overline{F M}_{n, i}^{1}$ where $\bar{R}_{n, i+1}$ appears in the difference quotient. The definitions of the approximating source terms etc. at the $i$-th time step are completely analogous to the 
definitions of these quantities at time $t=i \tau$ in case of the phase space discretization. For example, for $r>0$,

$$
\bar{\rho}_{i}(r):=\frac{1}{4 \pi r^{2} \delta} \sum_{n=1}^{N} \bar{M}_{n, i} \bar{E}_{n, i} \chi_{\delta}\left(r-\bar{R}_{n, i}\right),
$$

and analogously for $\bar{p}_{i}$ and $\bar{\jmath}_{i}$. Inserting these into the formulas for $\bar{m}(t), \bar{\lambda}(t)$, and $\bar{\mu}(t)$ defines corresponding approximations at the $i$-th time step of these quantities.

To measure the errors for the fully discretized system we define

$$
\begin{aligned}
e_{i}^{R} & :=\max _{n \in \mathcal{N}, k \in\{0,1, \ldots, i\}}\left|\bar{R}_{n}\left(t_{k}\right)-\bar{R}_{n, k}\right|, \\
e_{i}^{W} & :=\max _{n \in \mathcal{N}, k \in\{0,1, \ldots, i\}}\left|\bar{W}_{n}\left(t_{k}\right)-\bar{W}_{n, k}\right|, \\
e_{i}^{M} & :=\max _{n \in \mathcal{N}, k \in\{0,1, \ldots, i\}} \varepsilon^{-3}\left|\bar{M}_{n}\left(t_{k}\right)-\bar{M}_{n, k}\right| ;
\end{aligned}
$$

note that these are monotonous in $i$. The convergence result for the fully discretized scheme is as follows:

Theorem 4.1 Suppose the assumptions of Theorem 2.9 hold, $\varepsilon \leq \delta^{2}$, and let $\delta$ and $\tau$ be sufficiently small. Then

$$
\begin{aligned}
& e_{i}^{R}+e_{i}^{W}+e_{i}^{M} \leq C \tau, \\
&\left\|\bar{m}(i \tau)-\bar{m}_{i}\right\|_{\infty}+\left\|\bar{\mu}(i \tau)-\bar{\mu}_{i}\right\|_{\infty}+\left\|\bar{\lambda}(i \tau)-\bar{\lambda}_{i}\right\|_{\infty} \leq C \tau, \\
&\left\|\bar{\rho}(i \tau)-\bar{\rho}_{i}\right\|_{\infty}+\left\|\bar{p}(i \tau)-\bar{p}_{i}\right\|_{\infty}+\left\|\bar{\jmath}(i \tau)-\bar{\jmath}_{i}\right\|_{\infty} \leq C \frac{\tau}{\delta}
\end{aligned}
$$

for all $i \in \mathbb{N}$ such that $i \tau \in[0, T]$, the time interval on which the solution $f$ is to be approximated.

As to the proof only some indications are given since once the fully discretized system is set up in the appropriate way, what remains is to establish a series of auxiliary results most of which have analogues in the semi-discretized approximation which were proven in that situation. The detailed proof can be found in 21]. Let

$$
J_{\varepsilon, \delta}:=\left\{i \in \mathbb{N} \mid i \tau \in\left[0, T_{\varepsilon, \delta}\right]\right\} .
$$

The approximations are calculated as long as $i \in J_{\varepsilon, \delta}$ and for all $n \in \mathcal{N}$ and $k \in\{0, \ldots, i\}$ the estimates

$$
\varepsilon^{-3} \bar{M}_{n, k},\left|\bar{W}_{n, k}\right|, \bar{R}_{n, k}, \frac{1}{\bar{R}_{n, k}},\left\|e^{2 \bar{\lambda}_{k}}\right\|_{\infty},\left\|\bar{\rho}_{k}\right\|_{\infty} \leq 4 D
$$

hold; observe the analogy to (3.1), (3.3). The set of all these indices $i$ is denoted by $J_{\varepsilon, \delta, \tau}$ For these $i$ the error estimates from Theorem 4.1 are established. This is then used to show that if $i \in J_{\varepsilon, \delta, \tau}$ and $i+1 \in J_{\varepsilon, \delta}$ then $i+1 \in J_{\varepsilon, \delta, \tau}$, provided 
the time step $\tau$ is sufficiently small. Since $T_{\varepsilon, \delta}=T$ for sufficiently small $\delta$ this completes this indication of the proof.

Acknowledgment: GR acknowledges support by the Wittgenstein 2000 Award of P. A. Markowich, funded by the Austrian FWF.

\section{References}

[1] H. AndrÉasson. The Einstein-Vlasov system/Kinetic theory. Living Reviews in Relativity, to appear

[2] C. K. Birdsall, A. B. Langdon. Plasma Physics via Computer Simulation. McGraw-Hill (1985).

[3] J. M. Dawson. Particle simulation of plasmas. Rev. Modern Phys. 55 (1983), 403-447.

[4] K. Ganguly, J. T. Lee, H. D. Victory, JR. On simulation methods for Vlasov-Poisson systems with particles initially asymptotically distributed. SIAM J. Numer. Anal. 28 (1991), 1574-1609.

[5] K. Ganguly, H. D. Victory, JR. On the convergence of particle methods for multidimensional Vlasov-Poisson systems. SIAM J. Numer. Anal. 26 (1989), 249-288 .

[6] R. Glassey, J. Schaeffer. Convergence of a particle method for the relativistic Vlasov-Maxwell system. SIAM J. Numer. Anal. 28, 1-25 (1991).

[7] P.-L. Lions, B. Perthame. Propagation of moments and regularity for the 3-dimensional Vlasov-Poisson system. Invent. Math. 105 (1991), 415430 .

[8] I. Olabarrieta, M. W. Choptuik. Critical phenomena at the threshold of black hole formation for collisionless matter in spherical symmetry. Phys. Rev. D. 65 (2002), 024007

[9] K. Pfaffelmoser. Global classical solutions of the Vlasov-Poisson system in three dimensions for general initial data. J. Diff. Eqns. 95 (1992), 281303.

[10] G. REIN. Static solutions of the spherically symmetric Vlasov-Einstein system. Math. Proc. Camb. Phil. Soc. 115 (1994), 559-570.

[11] G. ReIn. The Vlasov-Einstein System with Surface Symmetry. Habilitationsschrift, Munich, 1995.

[12] G. ReIn. Static shells for the Vlasov-Poisson and Vlasov-Einstein systems. Indiana University Math. J. 48 (1999), 335-346. 
[13] G. Rein, A. D. Rendall. Global existence of solutions of the spherically symmetric Vlasov-Einstein system with small initial data. Commun. Math. Phys. 150 (1992), 561-583.

[14] G. Rein, A. D. Rendall. The Newtonian limit of the spherically symmetric Vlasov-Einstein system. Commun. Math. Phys. 150 (1992), 585-591

[15] G. Rein, A. D. Rendall. Smooth static solutions of the spherically symmetric Vlasov-Einstein system. Ann. de l'Inst. H. Poincaré, Physique Théorique 59 (1993), 383-397.

[16] G. Rein, A. D. Rendall. Compact support of spherically symmetric equilibria in non-relativistic and relativistic galactic dynamics. Math. Proc. Camb. Phil. Soc. 128 (2000), 363-380.

[17] G. Rein, A. D. Rendall, J. Schaeffer. A regularity theorem for the spherically symmetric Vlasov-Einstein system. Commun. Math. Phys. 168 (1995), 467-478.

[18] G. Rein, A. Rendall, J. Schaeffer. Critical collapse of collisionless matter: A numerical investigation. Phys. Rev. D 58 (1998), 044007.

[19] A. D. Rendall. Cosmic censorship and the Vlasov equation. Class. Quantum Grav. 9 (1992), L99-L104 .

[20] A. D. Rendall. The Newtonian limit for asymptotically flat solutions of the Vlasov-Einstein system. Commun. Math. Phys. 163 (1994), 89-112

[21] T. Rodewis. Numerical treatment of the symmetric Vlasov-Poisson and Vlasov-Einstein system by particle methods. PhD thesis, Munich 1999.

[22] J. Schaeffer. Discrete Approximation of the Poisson-Vlasov System. Q. Appl. Math. 45 (1987), 59-73.

[23] J. Schaeffer. Global existence of smooth solutions to the Vlasov-Poisson system in three dimensions. Commun. Part. Diff. Eqns. 16 (1991), 13131335 .

[24] S. L. Shapiro, S. A. Teukolsky. Relativistic Stellar Dynamics on the computer. I Motivation and numerical method. Astrophys. J. 298 (1985), $34-57$.

[25] S. L. Shapiro, S. A. Teukolsky. Relativistic Stellar Dynamics on the computer. II Physical applications. Astrophys. J. 298 (1985), 58-79.

[26] S. L. Shapiro, S. A. Teukolsky. Relativistic Stellar Dynamics on the computer. III. Astrophys. J. (Letters) 292 (1985), L 41.

[27] S. L. Shapiro, S. A. Teukolsky. Relativistic Stellar Dynamics on the computer. IV Collapse of a stellar cluster to a black hole. Astrophys. J. 307 (1986), 575-592. 
[28] H. D. Victory, JR., G. Tucker, K. Ganguly. The convergence analysis of fully discretized particle methods for solving Vlasov-Poisson systems. SIAM J. Numer. Anal. 28 (1991), 955-989.

[29] H. D. Victory, JR., E. J. Allen. The convergence theory of particle-incell methods for multidimensional Vlasov-Poisson systems. SIAM J. Numer. Anal. 28 (1991), 1207-1241. 